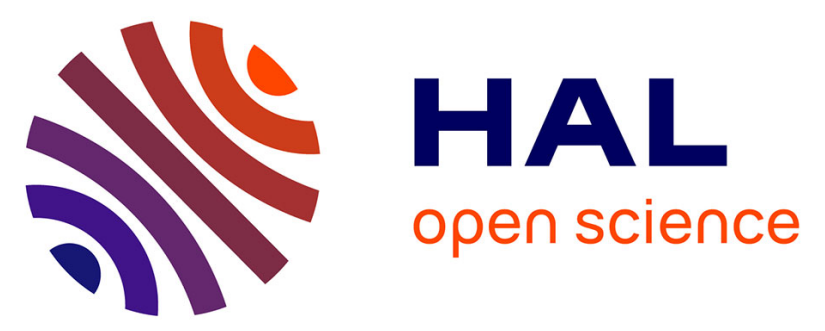

\title{
Review of Clinical and Technological Consideration for MRI-guided Robotic Prostate Brachytherapy
}

Sepaldeep Singh Dhaliwal, Taha Chettibi, Sarah Wilby, Wojciech Polak, Antony L. Palmer, Nick Reynaert, Rochdi Merzouki

\section{- To cite this version:}

Sepaldeep Singh Dhaliwal, Taha Chettibi, Sarah Wilby, Wojciech Polak, Antony L. Palmer, et al.. Review of Clinical and Technological Consideration for MRI-guided Robotic Prostate Brachytherapy. IEEE Transactions on Medical Robotics and Bionics, 2021, 3 (3), pp.583-605. 10.1109/TMRB.2021.3097127 . hal-03291021

\section{HAL Id: hal-03291021 \\ https://hal.science/hal-03291021}

Submitted on 18 Oct 2021

HAL is a multi-disciplinary open access archive for the deposit and dissemination of scientific research documents, whether they are published or not. The documents may come from teaching and research institutions in France or abroad, or from public or private research centers.
L'archive ouverte pluridisciplinaire HAL, est destinée au dépôt et à la diffusion de documents scientifiques de niveau recherche, publiés ou non, émanant des établissements d'enseignement et de recherche français ou étrangers, des laboratoires publics ou privés.

\section{(ㄷ)(1) $\$$}

Distributed under a Creative Commons Attribution - NonCommercial| 4.0 International 


\title{
Review of Clinical and Technological Consideration for MRI-guided Robotic Prostate Brachytherapy
}

\author{
Sepaldeep Singh Dhaliwal (D), Taha Chettibi D, Sarah Wilby, Wojciech Polak, Antony L. Palmer (D), Nick \\ Reynaert (D), and Rochdi Merzouki (D)
}

\begin{abstract}
Low Dose Rate Brachytherapy (LDR-BT) is a technique for treating localized prostate cancer by implanting radioactive seeds. In conventional practice, the delivery of seeds is performed using transrectal ultrasonography (TRUS) imaging for implant guidance and checked using computed- tomography for post-implant dosimetry. In the case of TRUS, accuracy can be compromised due to sub-optimal imaging. Magnetic Resonance Imaging (MRI), however, is known to provide better soft-tissue contrast, therefore, increasing the ability to detect small lesions; for that reason, the integration of intraoperative MRI in BT workflows has been investigated over the last two decades. The fusion of preoperative MR-images during TRUS-brachytherapy is possible. However, the image registration process introduces a source of uncertainty. Manual, real-time intra-operative LDR-BT is challenging under MRI due to confined space and procedural workflows. This motivates the development of MRI-compatible robots for prostate BT, with potential advantages of improved source placement accuracy and final dosimetry. In this paper, the state-of-art of technological components in MRI compatible robots, especially for LDR-BT, has been presented. This systematic review helps us to position an ongoing Cooperative Brachytherapy project, developing a real-time MRI-guided robot for adaptive LDR-BT. The design approach includes integrating separate modules: imaging, dose planning, needles, and robot.
\end{abstract}

Index Terms-Focal therapy, MRI Robot, Prostate cancer, Prostate intervention, Robotized Prostate Brachytherapy.

\section{INTRODUCTION}

$\mathbf{P}$ ROSTATE cancer is one of the most common male cancers, particularly for men over 50. More than 1.2 million new cases of prostate cancer were diagnosed across the world in 20181

In the case of prostate cancer treatment, radiotherapy is common practice. Radiotherapy can be delivered in two ways:External beam radiation therapy (EBRT) and Brachytherapy

This work has been co-funded by the European regional development Fund under EU Interreg 2 Seas, Grant CoBra: 2S04-022 (Corresponding author: Sepaldeep Singh Dhaliwal)

Sepaldeep Singh Dhaliwal and Rochdi Merzouki are with Centre de Recherche en Informatique, Signal et Automatique de Lille (CRIStAL) UMR CNRS 9189, University of Lille, 59650 Villeneuve d'Ascq, France (e-mail: [sdhaliwal; rochdi.merzouki]@ univ-lille.fr).

Taha Chettibi is with the Mechanical engineering Department, University of Blida1, Algeria, and was a visiting professor at CRIStAL, University of Lille, 59650 Villeneuve d'Ascq, France (e-mail: tahachettibi@gmail.com).

Sarah Wilby, Wojciech Polak, and Antony Palmer are with Medical Physics Department, Portsmouth Hospitals NHS Trust, UK (e-mail: [sarah.wilby; wojciech.polak; antony.palmer]@ porthosp.nhs.uk).

Nick Reynaert is with Medical Physics Department, Oscar Lambert, Lille, FR; ULB, Brussels \& Institut Jules Bordet, Brussels, BE (e-mail: Nick.Reynaert@bordet.be)

${ }^{1}$ https://gco.iarc.fr/tomorrow/en
(BT). BT can be carried out as Low Dose Rate (LDR) or High Dose Rate (HDR).

LDR-BT uses permanent placement of many radioactive seeds, isotopes I-125, Pd-103 or Cs-131, compared to HDRBT, which uses a single Ir-192 or Co-60 source wire inserted and retracted back to the afterloader after specific dwell positions and times. Prostate BT is lower cost than alternative treatments of surgery and EBRT, and has equivalent or reduced complications [1]. BT is common practice for prostate cancer treatment and in routine use utilizing transrectal ultrasonography (TRUS) as image-guidance modality. MRI based BT is under clinical investigation.

The most commonly available imaging modality at clinical sites are TRUS and computed-tomography (CT) for prostate BT. There are advantages and disadvantages associated with each imaging modality to adapt it for clinical use [1]. As TRUS provides a visualization of prostate motion during needle insertion but has lower tissue contrast as compared to CT or MRI. CT is capable of faster image acquisition but it does not provide good image resolution of base and apex of prostate, and volume-metric assessment is also inferior to MRI. MRI is capable of multi-parametric imaging sequences such as $\mathrm{T} 1-$ weighted $(\mathrm{T} 1-\mathrm{w})$ - morphological information and contrast fatty tissues and $\mathrm{T} 2-$ weighted $(\mathrm{T} 2-\mathrm{w})$ - zonal anatomy along with edema and inflammation. MRI can be integrated into treatment planning. MRI has restrictions with MR-compatibility and safety of BT-module and its components, limited availability of MRI for imaging as in high demand within hospitals, and in-bore workspace constraints. Currently, MRI use for seed placement is not common and does need strict safety protocols for high-field (3 Tesla) MRI. The visibility of seeds is also challenging under MRI as they appear voids and can be confusing with the needle tracks.

Taking an account of BT treatment, it is multi-component treatment process involving integration of - Imaging, dose planning, Needle/applicator, prostate anatomy, and seed (in case of LDR-BT). Based on the prostate anatomy being softtissue and its motion during needle insertion, makes challenging to deliver dose to distorted or shifted target sites and the same time accounting for the safety of organs at risk (OARs). Dose calculations with respect to the deformed, shifted, or damaged tissue poses another challenge for precise dose delivery. Thus current clinical investigations are focusing on adaptive dose calculation and dose delivery intraoperatively under real-time MR-imaging. To avoid organ deformations the concept of adaptive dose calculation and delivery motivates 
the clinicians to minimize the needle punctures to reduce tissue damage and edema to the organ. It is also intended to reduce the procedural time and improve targeting accuracy which ultimately helps in minimizing the number of needle reinsertion for the same target site. Referring to aforementioned challenges to increase the accuracy of seed delivery and needle placement, the robotized concepts and solutions are developed and being investigated with a different imaging modality, reviewed in [2].

Since the application of MRI in radiotherapy is under clinical research at rapid rate due to its precision and its increasing availability, particularly for BT [3], [4]. MRI provides superior discrimination of intraprostatic lesions and OARs critical in radiation oncology for improved target localization and accurate treatment planning.

In parallel, MR-robot developments are also an active area of research for intraoperative seed deposition/HDR-implant to take full advantage of MRI for accurate dose delivery. The delivery of radioactive seeds/HDR-implant to targeted lesion sites rather the potential for improved geographic accuracy of seed placement could lead to an improved accuracy and confidence in focal brachytherapy.

This paper compares the developed MRI-guided robotized systems for prostate LDR-BT and MRI robot-guides for prostate needle placement that have potential extension towards LDR-BT. The preliminary analysis of existing systems identified four main interacting modules within their architectures Fig 1, namely: an imaging system, a dosimetric treatment planning system (TPS), a needle trajectory planning software, and an MR-compatible robot. These modules might be connected differently according to the adopted workflow. Nowadays, robotic systems are increasingly complex, not only

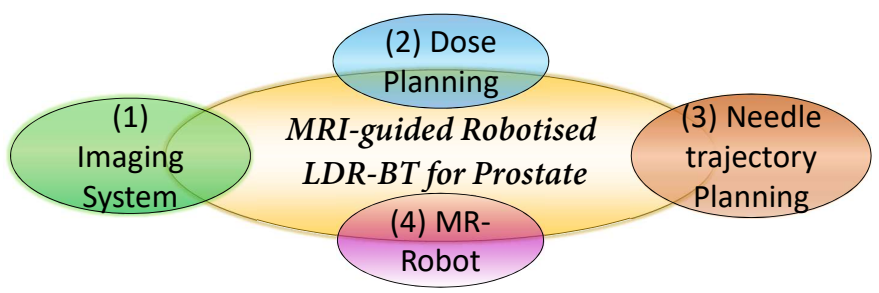

Fig. 1. System of Components System for Robotic LDR-BT of the Pr Cancer.

by their monolithic configuration, but also by their operal in a variable and complex environment. Thus, these engi: ing systems, which can cooperate to perform global mis: and can adapt to changes in their surrounding environr can be described in the form of a concept known as Sy of Component Systems (SoCS) [5].

According to Fig,1. MRI-guided robotic LDR-BT for prostate is a set of physical and biological systems composed of: MRI scan, MRI-based robot guide, intervention module for biopsy or brachytherapy and patient. All of these systems are independent operationally and managerially; they are not connected physically but communicate with each other in order to realize a common mission, which concerns a robot-guided intervention.
For example, in an LDR-BT workflow, the starting point is acquiring a set of images ( TRUS, CT, or MRI) of the required anatomical volume using the imaging system (1). It permits contouring of the appropriate regions of interest and OARs on the images and subsequent reconstruction of $3 \mathrm{D}$ virtual models from the contours. These images and built virtual models are then communicated to a dose planning system (2), which in conjunction with the needle trajectory planning system (3), optimizes the needle access points, needle trajectory path, and source placement, with dose calculation to optimize source position. Finally, the MR-robot (4) executes the control scenario for trajectory tracking of the needle's tip, reaching the tissue targets with the required accuracy for seed deposition.

It is hypothesized that a modular system for robotized LDRBT will bring the following benefits:

1) Improved implant quality.

2) Reduced operator-dependent uncertainty, and reduced BT technique 'learning curve'.

3) Ability to treat higher-complexity cases.

This paper reviews the robotic developments in the last 15 years targeting MRI compatibility used for prostate cancer treatment under MRI. Two broad reviews exist on MRIrobotic developments namely, Tsekos et al. [6] in 2007 and Monfaredi et al. [7] in 2018, and report of task group 192 on guidelines for image-guided robotic-BT in 2014 [2]. In the present work, a focus on prostate BT under MRI and potential robots with prostate-intervention towards brachytherapy under MRI is considered, it extends the broader comparison based on treatment concept, robot design and control, needle specifications and control, imaging, dosimetry, and inclusion of recent developments of MRI-robots have been made till the year 2020. Fig 2 illustrates the methodology of selection of research articles for review based on PRISMA [8]. The objective is to present information on recent developments of MRI-robots for prostate intervention and related modules which can be used to form an integrated complete solution for automated prostate brachytherapy under MRI-guidance.

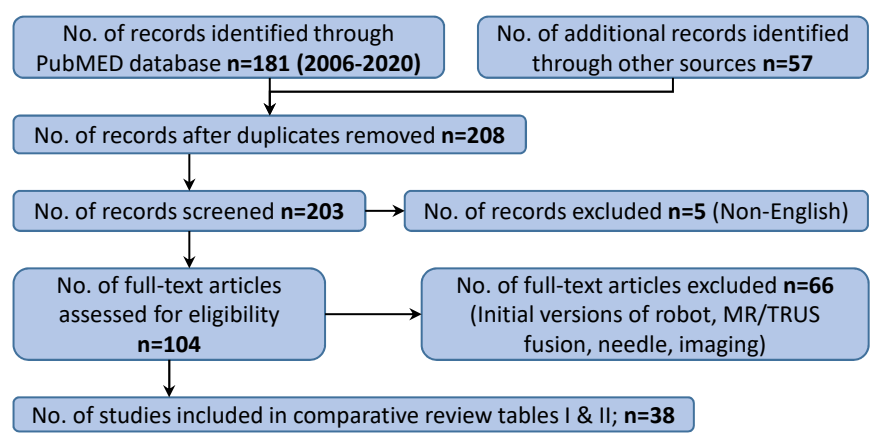

Fig. 2. The flowchart for the identification of eligible articles for review.

The survey was adopted with PubMed.gov database using keywords - (("MR-guided" OR MRI OR "Magnetic resonance") AND (Robot OR robotic OR robotized OR robotised) AND (Prostate OR Prostatic) AND (Brachytherapy OR Biopsy OR intervention)) NOT (prostatectomy[Title/Abstract]); resulting in a total of 181 papers (2006-2020) and additional 57 
papers were identified through IEEE Xplore and citations. Only full-texts in English were selected for reviewing title and abstract, papers related to High-intensity focused ultrasound (HIFU), US, and Laparoscopy were excluded. The papers were further shortlisted based on research groups and considering the recent publication of robotic developments and demonstrations of same robotic system for review tables I and II]

The paper is structured as follows: Section II discusses MRI and robotics in prostate brachytherapy. Section III presents MRI-guided robot for prostate intervention, section [V] is about imaging and processing, and section $\mathrm{V}$ discusses about dosimetry. Section $\mathrm{VI}$ and $\mathrm{VII}$ are respectively about discussion and conclusion in the domain of robotized MRI-guided prostate treatment concepts.

\section{RoBOtICS AND MRI IN PROSTATE BRACHYTHERAPY}

\section{A. Robotics in LDR Prostate BT}

Demand for surgical robots in radiotherapy is driven by the need to improve seed delivery accuracy, reduce operatordependence on quality, and prevent medical staff from radiation exposure. In contrast, manual LDR-BT techniques, with a rigid template, resulting in approximately 3-6 $\mathrm{mm}$ seed placement accuracy in-vivo [2]. The standard specifications of an image-guided robotic-BT should achieve a spatial accuracy of $1.0 \mathrm{~mm}(\mathrm{SD}= \pm 0.5 \mathrm{~mm}$ ) for seed placement within test phantom conditions and 0-2 $\mathrm{mm}$ under in-vivo conditions [2].

The robotized concept should cater to all desired operations to replace the manual procedure. However, it is not easy to define a single workflow of robotized LDR-BT, which depends on autonomy [2], the involvement of different imaging modalities, and the robot's design functionalities. For example, Fig 3 shows the detailed robotized workflow's main stages defined in [2]. From the next section onward, we will discuss the robots only in the context of MRI-guidance.

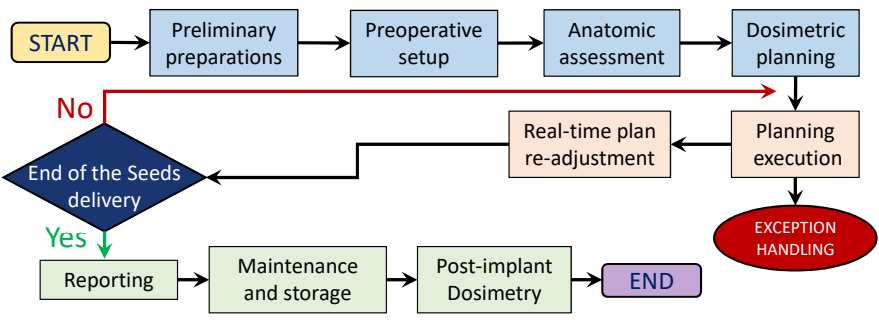

Fig. 3. Clinically specific workflows for robotized prostate LDR-BT.

\section{B. MRI in Prostate Brachytherapy}

The MRI technique is commonly used in diagnosing and staging prostate cancer, offering good soft-tissue contrast with non-invasive visualization of pelvic anatomy. The incorporation of Dynamic Contrast Enhancement (DCE) and Diffusion Weighted Imaging (DWI) added to T2-W MRI; results in improved detection of lesions [9]. The MRI images are potentially helpful in limiting dose to OARs while still delivering precise radiation treatment planning with improved clinical outcomes [10].
Advances in MRI have enabled its integration at different BT stages, briefed in II-C. Due to MRI's high soft-tissue contrast with an option to attain different MRI-sequences, e.g., T1-w, T2-w, DWI, etc. MRI is currently being practiced under clinical investigations and treatment for TRUS + MRI fusion-based BT [11] and direct MRI-based BT [12], [13]. Clinical outcomes under direct MRI based BT treatment motivated the feasibility of MRI based robotic BT-solutions for precise and accurate placement of radioactive seeds.

Unfortunately, MRI has its own limitations, such as restricted in-bore workspace, material compatibility, and MRI safety. It makes the development of MRI-guided interventional robots a challenging task. Another challenge with MRI scanners is their limited availability ${ }^{2}$ and most of the institutions have diagnostic scanners with vendor-specific sequences and demand sequence optimizations, and scanners required to be adapted or dedicated scanners needed for any robotized realtime prostate interventions for such treatment. During the last two decades, numerous teams have developed MRI-robot prototypes to handle several tasks inherent to prostate cancer treatment. Often for these prototypes, the interventional tasks take place outside the MR-scanner. In the case of LDR-BT, the needles' insertion takes place outside the MRI bore and is validated by a new MRI-scan, which lacks intraoperative anatomic information and any target shift in soft-tissues during needle insertion. This can reduce the geographical accuracy of implantation. It is preferable to perform intraoperative insertions inside the MRI with real-time image guidance. Ways of adapting the workflow in Fig 3 to include MRI are discussed in the next sub-section.

\section{Stages of MRI incorporation for LDR Prostate BT}

MR-imaging can be incorporated into prostate BT at different stages of clinical workflows. Wang et al. [4] divided BT workflow into five phases: diagnosis, treatment planning, implantation (needle placement), verification, and delivery. Fig 4 displays an example of different workflows incorporating MRI for LDR-BT, described below.

1) MRI-informed BT: MRI is used for diagnosis, and the information provided by MRI can be used directly or indirectly for optimal needle placement. It also includes the usage of MRI for post-implant dosimetry. Act of seed delivery takes place out of bore after needle tip verification.

2) MRI-Based BT planning: MRI is used to delineate targets and normal tissues for treatment planning only. This works as dual-modality planning for LDR-BT using the ultrasonography (US) acquired data for treatment planning, co-registering the MRI data for structural delineation. MRI-based contouring and treatment planning for seed delivery can also be attempted without US [14].

3) MRI-guided BT implants: MRI guides the physical insertion of needles for optimal placement [15]. Robots using MRI to guide the needle placement would come under this workflow.

${ }^{2}$ https://ec.europa.eu/eurostat/fr/web/products-eurostat-news/-/DDN20200724-1 


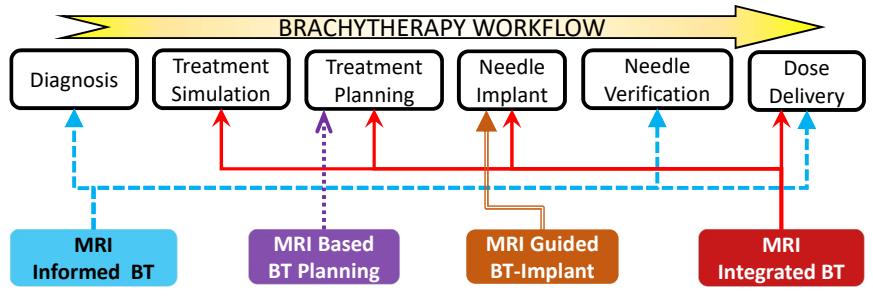

Fig. 4. Clinically specific workflows for prostate LDR-BT incorporating MRI, arrows connecting the stage using MR-imaging for LDR-BT.

4) MRI-Integrated BT: MRI is used for treatment planning, needle implantation, and verification.

Most of the robotic developments use MRI for needle placement, falling under MRI-guided BT implant's workflow. For this workflow, it is necessary to move the patient out of the scanner bore for confirmation or verification of needle tip location, with respect to target sites, followed by manual insertion by the Physician. Whereas, BT using real-time MRI for insertion guidance would fall under the MRI-integrated $B T$ workflow. In this workflow, the BT-system must be MRSafe or MR-Conditional to place it at a specific distance from the MRI bore. MRI-robots using intraoperative real-time image guidance for in-bore seed delivery should fall under the MRI-integrated BT category. In both cases, it requires patient immobilization to minimize any anatomical changes while the patient's ingress-egress to the scanner during needletip position verification. The workflows discussed above help explore the scope of incorporating MRI to perform robotized real-time intra-operative prostate BT treatment. The following sections review the MR-based prostate treatment concepts, components, and critical requirements.

\section{DESIGN CONSIDERATIONS FOR MRI-GUIDED Robot DEVElopMEnt TOWARDS PROSTATE INTERVENTION}

This section discusses and reviews the robotized prostate interventions, with a prime focus on prostate LDR-BT MRrobots and potential MR robot-guides for seed deposition. The MR-biopsy robots have only been studied for their application towards LDR-BT; as needle placement concepts for prostate under MRI. Diagnosis and treatment concepts are explored in III-A, practice in previous developments along with their robot design and control in III-B prostate intervention needles are reviewed in III-C, and the MR-robots are reviewed in III-E

\section{A. Diagnosis and treatment concepts}

This subsection discusses the robotized MR-operations for both diagnosis and treatment. The critical components and clinical procedure's building blocks to be considered are the type of MR-scanner, prostate access path, patient placement, different test conditions (in-vivo, ex-vivo or in-vitro), rating the output at the Technology Readiness Level (TRL), and guided workflows.

1) MR based Prostate interventions: The needle placement interventions reviewed are listed below:

1) Biopsy (BX): BX is performed for diagnostic purposes; suspected lesion sites are targeted to remove tissue samples for further examination. D'Amico et al. [16] initiated manual MRI-guided prostate BX and evolution towards robotized MRI-guided prostate BX in [17] .

2) LDR-BT: Uses radioactive seeds (isotopes I-125, Pd103 or historically Cs-131) encased in titanium, called LDR seeds. These seeds are delivered permanently to the prostate according to a treatment plan which describes the geographic locations for a desired dosimetric target coverage.

3) Marker deposition (MD): The gold-markers are deposited inside the prostate for EBRT. The robots used for markers deposition are considered in this review, as the concept is similar to seed placement [18].

4) Ablation (Ab): In ablation high-energy laser is used to heat rapidly the target site resulting in cell death [19].

In the literature, it was found that most robot-based developments are dedicated to BX rather than BT. The reason fewer MRI-robots have been developed for LDR-BT can be understood as increased complexity in the accuracy required in BT for seed delivery in 3D space. In contrast, it is not the case for the $\mathrm{BX}$ where high accuracy is required in the axial plane, but there is a lesser requirement on accuracy in depth. This is because an approximately $2 \mathrm{~cm}$ length of the core is extracted, only part of which needs to traverse the lesion Further, BX's time procedure is 10 minutes with local anesthetics and BT's minimum 45 minutes with general anesthetics. Due to procedure time constraints, there are fewer scanners as compared to the number of patients.

2) MRI Scanner and the role of field strength: MRI scanners can be classified based on the type of bore (closed or open) or can be related to their field strength Tesla (T). Open bore scanners are generally less than $1 \mathrm{~T}$, whereas closed bore scanners are usually of 1.5-3 $\mathrm{T}$, with an in-bore radius around $60 \mathrm{~cm}$ or $70 \mathrm{~cm}$ (wide-bore).

Closed bore scanners are preferred over open due to their imaging quality. However, closed bore MRI has limited workspace to integrate a robotic concept for prostate treatment. It is essential to considered field strength as it influences signal-to-noise (SNR) ratio, geometric-distortions (GD), and artifacts in imaging. It is recommended to target minimal GD of the prostate for MRI-guided LDR-BT [4]. $3 \mathrm{~T}$ scanners generate high-resolution images, improving tissues' delineations for dose delivery, especially for intraprostatic tumor dosage boost. However, MR-compatible needles (e.g., titanium) generate significant artifacts at $3 \mathrm{~T}$ field strength [4].

It is becoming increasingly common that radiotherapy centers to have dedicated MRI scanners as part of BT-suite for radiation oncology, or access diagnostic MRI scanners for brachytherapy workflows [20]. 1.5 T scanners can provide sufficiently improved visualization of prostate anatomy BT [4], and they have the advantage of fewer object-induced (metal seed or needle) artifacts. For robotic systems that include MRConditional components like sensors (discussed in III-B, the 1.5 $\mathrm{T}$ should be the preferred option with fewer restrictions with materials, resulting in low susceptibility artifacts compared to $3 \mathrm{~T}$. Also, $3 \mathrm{~T}$ scanners demand considerable sequence optimization for BT due to susceptibility issues [4]. Depending upon the availability of MR-scanner and safety protocols, it 
can be stated that a robotic system compatible with $3 \mathrm{~T}$ would also be compatible under $1.5 \mathrm{~T}$ scanners.

3) Patient Placement: Prostate access and patient positioning are important considerations in the designing and developing robots for MRI prostate intervention. The prostate can be accessed by (i) Transrectal (TR) technique, in which needles are inserted via the rectum and the patient is usually laid in a prone position, e.g., [21]. (ii) Transperineal (TP) technique, in which needles are inserted via the perineum, commonly used for TRUS and similarly preferred for MRI-guided prostate interventions, e.g., [22], [18]. (iii) Transgluteal (TG) technique, performed in cases when the rectal route of prostate examination and sampling is not available in patients with prior anorectal resection or other anal abnormality that precludes rectal access [23], e.g., [24]. (iv) Transurethral technique, in which a thin transurethral ablation applicator is inserted through the urethra towards the prostate with an endorectal cooling device 3 is not feasible for BT.

The TP approach is preferable and recommended over TR as it lowers the sepsis risk and offers more peripheral zone coverage of the prostate, around $98.5 \%$ with more accuracy. In contrast, TR covers only up to $64.9 \%$ [25].

The common patient positions for needle insertion to the prostate gland under MRI are left lateral decubitus (LLD) [22], [26], [27], Prone [21], Supine (SU) [18], [27] and Lithotomy (LT) [17], Semi-LT position opted in [28], [67]. Prone position has issues related to rectal gas, which may cause imaging artifacts. This position gives mixed reviews from experts and may be chosen due to MRI's confined space and provides easier access for the TR approach. The lithotomy position is commonly recommended to perform TRUS guided prostate BX and BT, offering better detection of lesion targets of deformable soft tissues than the prone or LLD position [29], resulting in less pain [58] accessing the prostate via the perineum. In addition, the prostate is more mobile under the decubitus position than SU or LT [25].

TP access is more convenient with the LT position. LT position can be achieved with the commercial existing MRSafe Uni-Lif ${ }^{5}$ specifically developed to stabilize the patient for MRI prostate intervention shown in [31]. Lastly, LT has an advantage with more workspace to target the TP area within the MRI.

4) Prostate position and targeting: The deformable nature of the prostate gland tissue, position surrounding the urethra, and the possibility of pubic arch interference (PAI), present challenges for interventional procedures.

According to data sets at Portsmouth Hospitals University NHS Trust, UK; prostate mean dimensions (with standard deviation) for length $50 \mathrm{~mm}(10 \mathrm{~mm})$, height $46 \mathrm{~mm}$ (11 $\mathrm{mm})$, width $48 \mathrm{~mm}(9.5 \mathrm{~mm})$, volume $63 \mathrm{~cm}^{3}\left(34 \mathrm{~cm}^{3}\right), \mathrm{n}$ $=238$. During the BT procedure, due to edema, the prostate can enlarge by up to $25 \%$, it can also rotate generally up to $14^{\circ}$ in coronal and $10^{\circ}$ in the sagittal plane [25]. It constitutes an additional consideration to be handled by any robotic system.

3 https://profoundmedical.com/new-tulsa/

4 https://www.alimed.com/lithotomy-patient-positioning-blog/

5 www.noras.de/en/mri-products/uni-lift-prostate-intervention-device/
Considering the different in-bore patient positions stated above, it is challenging for physicians to access the prostate due to the confined space and to perform MRI in-bore interventions. Out of bore interventions are therefore opted for with the aid of a compatible template but without realtime imaging, which compromises MRI's key advantage. For automated solutions, Song et al. [32] presented an Ultrasonic motor (USM) actuated template-guide for in-bore manual intervention, and Fischer et al. [33] increased the template size to $100 \times 100 \mathrm{~mm}$; however, it is still limited to manual needle placement. PAI can make it challenging to cover the prostate target with $100 \%$ of the prescription dose, and larger prostate sizes $\left(>55 \mathrm{~cm}^{3}\right)$ may not be appropriate for the BT treatment option when the angle of the needles are constrained by the external grid. Solution for such patients could be with oblique needle insertion or steerable needles.

Modern clinical procedures demand minimally invasive surgery, and robots have the potential to fulfill the minimal invasive surgery objective with minimal invasive needle insertion/placement (opted in [34]) using fewer entry points (to reduce trauma), without a template, and having the ability to perform oblique (non-parallel) insertions. This requires the robot to use a high level of image guidance to enable coverage of the whole prostate volume with better accuracy or with the potential for focal therapy.

Detailed workspace and prostate localization for transperineal needle path can be found in [35]. It can be stated that changes in leg positioning for in-bore intervention impact the prostate's anatomical positioning, but, in general, it was found through the review that TP is preferred for robotized concepts, with patients in the supine or lithotomy positions.

5) Test Conditions: It essential for the validation of any surgical device to demonstrate functional feasibility. Any robotic device for surgical intervention needs to perform accurate insertion according to the planned trajectory with precision. A robot needs to demonstrate feasibility under test conditions and pilot studies before any clinical application.

In previous developments of LDR-BT robots under MRI, tests were conducted with in-vitro [36], [37] conditions only, whereas for marker deposition (MD) and BX in-vivo [21], [22], [18], [38], in-vitro [67], [29] and ex-vivo [39] studies were also performed. Most of the existing robot developments have progressed to the needle positioning concept only, and physicians have to perform manual insertions. It is reviewed in section $\amalg I-E$

The testing conditions help to distinguish the systems based on TRL. TRL scale was introduced into the EU funded projects arena in 2014 to indicate a given technology's readiness. The TRL is scaled in nine level 6 . Most of the robot developments for prostate interventions are up to TRL scale 4 only (Technology validated in a lab).

6) Guided workflows: MRI with the potential of interactive intra-operative guidance offers the advantage of localized target accuracy [40] and needle navigation over the traditional guidance using pre-operative images. Performing minimally

6 https://enspire.science/trl-scale-horizon-2020-erc-explained/ 
invasive BT intraoperatively with MRI comes under the subject of interventional radiology.

Currently, in the BX procedure, pre-operative images are used for planning in which fusion errors may occur due to a mismatch of patient positioning or organ movement.

To overcome such errors, MRI can be incorporated with LDR-BT to use it as a real-time image-guidance is discussed above in II-C Essential phases involved are lesion localization, needle placement, treatment planning, and needle tip verification. In most of the reviewed robot prototypes which are developed to perform prostate interventions under realtime MRI-guidance, significant developments are still limited to needle placement only and that too, with manual needle insertion (BX or Seed delivery). Only a few robot development projects achieved an image-guided workflow that do not move patients out of the MRI scanner for needle insertion exists; these are summarized in Table [

Direct MRI guidance could help robot scanner registration. Stoianovici et al. [41] demonstrated this using a long registration marker under 3D T1-w imaging sequence and tissue targeting under $\mathrm{T} 2-\mathrm{w}$, recommended to keep the same frequencyencoding direction in both scans. Geometric corrections can be achieved under $\mathrm{T} 2-\mathrm{w}$ using 'distortion correction' [41]. Moreira et al. [42] demonstrated teleoperated needle insertion under real-time MRI.

We divided the intraoperative procedure taking place inside the scanner room into two categories: (1) In-bore needle insertion (prostate intervention) under real-time MRI guidance as RT-intraoperative. (2) Out of bore needle guide insertion with in-bore position verification (moving patient in and out scanner between these two stages), referring to it as postimplant needle verification (PINV). The latter is adopted in the majority of developments with needle-guide positioning and needle tip verification, which requires a new scan for needle insertion, listed in Table I

\section{B. MRI Robot Design and Control}

This section details the type of actuators (III-B2) and sensors used (III-B3). Table discussing their MR-compatibility and the existing prostate intervention robots. A surgical robot is required to attain device regulatory and safety clearances before commissioning for any surgical interventions fulfilling the needle intervention requirements. For any BT-robot for MRI-environment, the robot is desired to be compact in design for in-bore prostate intervention. The robot must achieve needle-placement accuracy of $0-2 \mathrm{~mm}$ and able to generate around 30 to $50 \mathrm{~N}$ force considering factors of safety and reaction forces for perineum skin and prostate puncture based on the recommendation by Podder et al. [2]. The robot should be capable of performing oblique insertions to target occluded regions of the prostate (such as behind PAI for $>60 \mathrm{~cm}^{3}$ prostate), so it is desired for any robot to have a minimum of 5 DOF. A modular design approach should be targeted with an option to integrate different modules on the same robot guide so that it can be adapted for BX, LDR-BT, or HDR-BT modules.
1) MR Compatibility, Safety, and Materials: MRI is subject to specific safety requirements compared to US. The three fundamental features of MRI that are of concern when developing any MR compatible equipment are high static magnetic field strength, fast switching magnetic gradients, and radio-frequency pulses. The extreme magnetic-field conditions of MRI necessitate robot development with MR compatible materials, actuators, sensors, and shielded electronics.

MRI is very sensitive to electromagnetic interference (EMI). Equipment and mechatronic devices placed inside the scanner room need to be well shielded to avoid any EMI. Fast shifting gradient magnetic fields can result in electric fields and eddycurrents in conductive materials, affecting the magnetic field's homogeneity, leading to image distortions [43]. These currents can cause heating of components during image acquisition; it necessitates to verify and test the same MR-sequences, which are to be opted for during clinical procedures [4]. The wiring of mechatronic devices coming into the scanner room can act as antenna radiating noise [44]. The ferromagnetic material components may result in hazardous projectiles and cause artifacts and distortions. Non-ferromagnetic, specific composite materials or plastics can be adopted after testing under MRI. Robot compatibility with MRI can be categorized as [45]:

1) The robot must be MRI-safe or MRI-conditional,

2) Robot does not cause any hazard to the patient or staff,

3) The robot does not affect the imaging quality of MRI when operational or powered off.

4) The functionality of the robot is not affected by the MRI magnetic fields.

Furthermore, for MR safe practices, the MRI scanner's region is divided into four zones [46]. Zone I: Freely accessible with lower magnetic field posing no risk to the general public with least restrictions. Zone II: Patients are under the supervision of MR staff, including the MRI screening room. Zone III: A restricted Zone where patients and staff are allowed after MRI screening only due to a detectable magnetic field. It is wellmarked with symbols and coded entrance. Zone IV: The room containing the MRI scanner and 5-Gauss line and prohibiting entry of any ferromagnetic component, e.g. implants, which can be affected by the strong magnetic field and potentially harm the patient.

In 2005 FDA revised MR device safety terminologies as MR-Safe, MR-Conditional, and MR-Unsafe refer Fig[5(A). MR-Safe devices are nonhazardous in all MRI zones. MR conditional items are deemed safe for specific MR-environments and conditions, depending upon the materials posing no harm or interference with imaging. These materials are prohibited from entering the bore and beyond the $20 \mathrm{mT}$ markings, allowing only after stringent testing under controlled conditions. MR-Unsafe items pose a risk under all MRI environments and cannot be allowed inside the MRI room. Reference of magnetic field intensity is shown in Fig 5(B). The MRrobot prototype should follow the ASTM standards (F2503, F2052, F2213, F2182, and F2119) [47], which are well described by Stoianovici et al. in [48]. According to F250313 , only non-conductive components come under the MR-Safe 


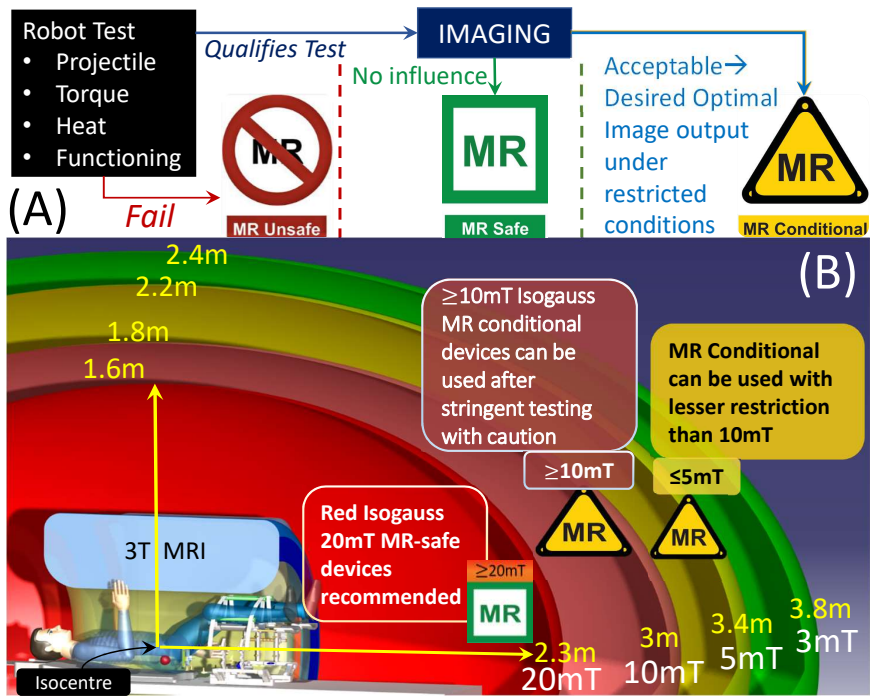

Fig. 5. (A) MRI compatibility classification (B) 3 T MRI Isogauss (parameter reference from $3 \mathrm{~T}$ Magnetom Prisma) and compatibility recommendations

category. Thus, most of the developments fall under MRConditional only. The reported developments used plastics like ULTEM, Teflon, PEEK [28], Delrin [49], acrylic, nylon [18], [49], Ertalon [50], Polyethylene terephthalate (PETE) [51], polyoxymethylene (POM) [52], high-modulus polyethylene (HMPE) and non-ferromagnetic metals like Aluminum, Titanium, Brass [49], Be-Cu [53], phosphor bronze [21] and ceramic materials. Some studies tested magnetic shields with permalloy and $\mathrm{Mu}$ metal up to $17 \mathrm{mT}$ [54] and EMF insulation cloths in-bore [55].

2) Actuation: Actuator selection for MR-robots, with desired torque and speed outputs of compact size, is challenging depending upon the functional complexities and robot architecture. To develop an efficient surgical LDR-BT robot, replacing the manual procedure desired clinical (in-vivo) accuracy is 0-2 $\mathrm{mm}$ for seed placement with a need to demonstrate spatial accuracy in controlled phantom conditions of $\leq 1.0 \mathrm{~mm}$ for seed placement [2].

Gassert et al. [57] described potential actuators for an MRI environment, including mechanical, pneumatic, hydraulic, piezo-motors, and electromagnetic (Lorentz actuators/ magneto-mechanical vibrotactile devices [60] and shielded dc motor). Some commercialized actuators have been tested by Fischer et al. [61]. In a study by Gassert et al. [57], wellshielded electromagnetic actuators (dc motors) were investigated within the MRI room at a distance of $\approx 2.5 \mathrm{~m}$ from the isocenter. Most of the developments focused on Piezo motors and pneumatic actuators (PN) [61]. Both have their pros and cons for robot development discussed in the next paragraphs.

PN devices do not produce image interference [62], making them an ideal candidate for MR-Safe robot development. On the other hand, they are bulkier, having controllability challenges with long pneumatic transmission lines due to delay response. On the other hand, they are bulkier, have control challenges due to long pneumatic transmission lines because of the delayed response. Additionally, the existence of inherent nonlinear friction force could result in difficulties in achieving the actuator's high spatial accuracy [63]. The issue related to time delay control is discussed in [30]. Thus the desired seed placement accuracy for LDR-BT is challenging with PN. Stoianovici et al. developed a novel pneumatic stepper motor PneuStep with $3.3^{\circ}$ step resolution [64] used for MR-Bot (MRSafe robot) [48] [65]; the motor is not commercialized.

Piezo motors have been a popular choice due to their compact size, high torque to weight ratio, high braking torque to lock position, inherent robustness, and scalability, making them advantageous over PN actuators. The compact size and accuracy advocate them for optimum size surgical robot development. Piezo motors can be classified into three types $i$ ) Piezoelectric motors (PZ) [Nonharmonic], ii) Piezo ultrasonic motors (USM) [Harmonic], and iii) Piezo inertia-motors. As piezo-motors are MR-Conditional may result in image noises and cause more image-noise due to their conventional motor drivers, methods to drive motor without noise interference are discussed in [61], [66]. Li et al. [37] studied PZ (nonharmonic), they observed that it has better noise suppression than USM (harmonic). Commercial existing harmonic motors are HR-2/4/8 $8^{7}$ (amplitude modulation), USR ${ }^{8}$ \& PUMRNM ${ }^{9}$ (frequency modulation), and non-harmonic are PiezoLeg $\$ 10$ (frequency modulation) [67].

The study by Fischer et al. [61] stated that Piezo-motors do not affect the MR images, and the source of noise is commercial motor drivers. In [67], the authors state that customized motor drivers generate signals via a direct digital synthesizer, a high-performance multi-channel digital-to-analog converter, and high power linear amplifiers can suppress image noise interference. Further, pi filters of high-efficiency remove highfrequency noises. Carvalho et al. [68] presented custom USM with reduced metallic content replacing the casing with ULTEM, a shaft of Derlin, and nylon screws, which resulted in lower distortions and better SNR.

Shokrollahi et al. [69], [70] tested USM inside the bore near isocentre under different placement configurations with normalized SNR outcomes to drop by $15-25 \%$ and found that parallel placement of USM to field resulted in more noise. Commercialized USMs' are MR-Conditional and can be used for robot development under controlled conditions (minimum $30 \mathrm{~cm}$ from MR-isocentre), targeting the least SNR losses with different configurations and power on/off mode. Other commercialized non-magnetic actuators can be used in future robotized concepts are Piezo-Sonic ${ }^{11}$ SCNUAA PMR $^{12}$, SQUIGGLE motor ${ }^{13}$ and Rotatex RUSR ${ }^{14}$

3) Sensors: Position sensing and force feedback are essential components for closed-loop control of any robot function. Surgical robots demand precise and reliable sensors. The conventional sensors adoption under MRI environment adds MR-

www.nanomotion.com/product-type/standard-motors/hr-series-motors/ 8 http://www.shinsei-motor.com/English/product/nonmagnetic.html chttps://piezotech.en.ec21.com/UltraSonic_Motor-1763572.html

10 www.Piezomotor.com

11 Www.piezo-sonic.com

12 http://en.scnuaa.com/nav/2.html

13 WwW.newscaletech.com

14 Www.beaconpipe.com 
compatibility restrictions. For MRI environments, the fiber optic-based sensors are suitable for position and force sensing.

Position sensing: Sensors are required for the position (lowlevel) control, e.g., potentiometers, optical encoders, laser, ultrasonic sensors, etc. Due to their material or electric components, many of them might not fit under MR-compatibility, inducing artifacts or image-distortions. Position sensing of joint space can be achieved by optical linear and rotary encoders, which calculates the end-effector position. Besides, under a controlled environment and conditions, researchers have used optical encoders and demonstrated their working feasibility under magnetic fields, discussed in the following paragraph.

MR-robots (Prostate intervention) incorporated commercial rotary (EM1-0-500-I) [67], linear (EM1-1-1250-I) [33] encoders and incremental quadrature encoder (E8P-512) [30] from US Digital ${ }^{\circledR} 15$ under $3 \mathrm{~T}$, with observations that artifacts are confined to $2-5 \mathrm{~mm}$ range close to encoder only. The standard US Digital optical encoders have $0.0127 \mathrm{~mm}$ linear and $0.072^{\circ}$ rotary resolution. Moreira et al. [71] used a reflective optical encoder (LIK41, Numerik Jena, tested up to 6 T magnetic fields ${ }^{16}$ for needle insertion depth measurement.

Some developments adopted self-made fiber optic sensor solutions in order to avoid compromise to imaging. The void of commercialized sensors or low-cost solutions were presented in the review [59]. Custom solutions can utilize reflective or transmissive optical encoders developed specifically for MRenvironment. Stoianovici et al. [64] adopted custom fiber optic quadrature encoders for Pneustep. The commercially available solution from Micronor Inc ${ }^{17}$ (MR-Safe) offers micro millimeter accuracy. The commercialized optical encoders' availability makes it feasible to adopt them for MR-robot position sensing.

Another method of position sensing and orientation based on only imaging is achieved with the aid of fiducial markers attached to the robot base or surgical tool/manipulator, e.g., Z-Frame, introduced in [72]. These can be of MR-spot or contrast agent filled solution tubes used for patient and robot registration.

In the case of HDR-BT, Borot de Battisti et al. [73] presented the HDR needle tracking based on Fiber Bragg gratings (FBG) sensors and reported an accuracy of $0.79 \mathrm{~mm}$ under RT-MRI tracking on prostate test-phantom.

Force sensing: Invasive task-space position feedback is critical to avoid inaccuracy resulting from tissue deformation and needle flexion. It can be achieved with strain-gauge and fiber-optic force sensors. Fiber-optic sensors are the preferred choice as they are inert to MR-environment. In contrast, straingauge sensors may result in electrical noise and complicated installation, causing more compatibility restrictions in MRfield.

Examples of some commercial sensors used are fiberoptic limit switches [32], piezo-resistive force feedback (FSS, SensoTechnics) [74], JR3 commercial force sensor for teleoperated breast biopsy [75], Piezo-proportional pressures valves

\footnotetext{
15 www.usdigital.com

16 https://www.motioncontroltips.com/?s=non+magnetic+encoders

17 https://micronor.com/products/rotary-encoders-mri/
}

by Hoerbiger-Origa (near MRI) [33], Custom Fabry-Perot interferometry (FPI) sensor based on FPI strain gauge by FISO Technologies Canada [76], [62], PX309-100GD5V pressuresensor (Omega, US) and Load cell MLP-10 by Transducer Techniques for force sensing used in [77]. In neurosurgery under MRI, a commercialized ATI-nano-17 force sensor was adopted for intra-operative surgery in a neuroArm robot [78]. Commercialized force sensors like FPI - FISO and ATI are solutions for MR-robot construction with target developments of TRL-6, though they are expensive but currently are the only on-self solution.

\section{Prostate Intervention Needle Control}

Needles are vital components directly interacting with the prostate gland. Clinical BT and BX needles are composed of: a cannula, a hollow tube, and stylet, a solid shaft. For BT, 18G needles are commonly used to insert stranded or loose seeds, which have an external diameter of typically $0.8 \mathrm{~mm}$ and a length of $4.5 \mathrm{~mm}$. Conventionally, straight bevel tip needles are used to target the prostate via TP using a template guide with holes $5 \mathrm{~mm}$ apart. Challenges associated with prostate motion and the target shifting during needle insertion are discussed by Stone et al. [79]. Dattoli et al. [80] demonstrated a prostate fixation technique in which motion can be restricted to 0.2 $m m$. Okamura et al. [81] studied diamond-tip needles, showing low resisting forces as compared to bevel-tip. Podder et al. [82] define needle (18G) insertion force requirements at TP skin of around $10 \mathrm{~N}$ and $8 \mathrm{~N}$ to puncture the prostate and to consider 3 to 5 folds for safety factors. Furthermore, the author stated that less axial force results in lower tissue deformation. In [83], the concept of needle rotation was introduced to reduce tissue trauma, and [84] presents needle angulation with the help of a template. Patriciu et al. [26] state fast-needle insertion for accuracy, and in contrast, Bosch et al. [18] recommend needle insertion in small steps to avoid prostate motion.

In consideration of the work mentioned above and to perform automated needle insertion in order to compensate for the target shift and needle deflection, active control needles can be achieved with the help of steerable needles. For example, a curvilinear approach [85], solutions based on bevel-tip or pre-bent needle tip steering ${ }^{18}$. Needle design aspects are not covered in detail in this paper. Since needle steering is an active research field with numerous developments and testing, the issues related to needle steering and control are well addressed in [86], and extensive review for needle guidance is presented by Kulkarni et al. [87]. A brief review has been considered and summarized in the portion of Table III of existing needle control practiced by research teams integrated to current MRI-robotized concepts.

Most of the institutes have tested straight insertions both manually [33], [39], [88] and automatic [37], [67] with robot-assisted needle placement. Robots with both straight and oblique needle insertions have been tested manually by [17], [29], [49], [89], and automatically by [22]. Trajectory planning concept of needle insertion during treatment has been demonstrated manually [17], [18], and automatically with a

\footnotetext{
18 https://cobra-2seas.eu/the-project/steerable-needles/
} 
physician defining the target [21], [18], [62], [55], [90], target + entry point [28] and target + insertion angle [29]. Currently, in clinical procedures, standard $18 \mathrm{G}$ (straight and bevel tip) needles are being used for both BT and BX in manual TRUS procedures and MR-compatible $18 \mathrm{G}$ (e.g., InvivoCorp. or Somatex) under an MRI environment. Concentric tubes [91] have been validated for neurosurgical treatment in simulation and phantom studies. Still, practicality is challenging in prostate BT due to the tube diameter, bending radius, material stiffness, and seed navigation feasibility. The robots based on MRI-BT treatment to cover whole prostate volume with the minimum number of insertions require the system to be without template and minimal insertions [34]. Indeed, it is needed to provide better needle insertion maneuverability, which further tackles the PAI and enhances the targeting accuracy.

1) MR-Needle Material: : Needle material composition is a crucial factor that needs to respect the MR-environment, structural rigidity for skin puncture, and remain inert to interacting biological tissues. So far, Ti or Ti-alloy needles are used for prostate intervention. Although Ti is considered an MRIcompatible material, it can still get heated due to RF-induced heating. Different scan parameters RF-power, altering electric field, resonance, and needle orientation, play a significant role in needle heating and safety [2]. The commercialized MRneedle like Invivo Corp. mentioned compatibility usage up to $1.5 \mathrm{~T}$ and recommended out-bore usage for $3 \mathrm{~T}$ [92]. Shorter wavelengths of $3 \mathrm{~T}$ cause local heating and larger artifacts [92]. Still, in case they pass artifact-image testing with $3 \mathrm{~T}$, they can be prone to thermal injuries near the isocentre and must be validated with thermal testing before any in-vivo setup. A recent study by Khodarahmi et al. [93] states MRConditional needles should be selected based upon optimized pulse sequence, needle length, and orientation (angle along the static magnetic field $\left.\left(B_{0}\right)\right)$. The study found a high-temperature rise when a needle is placed parallel to $B_{0}$, which may potentially cause tissue damage.

2) Needle Insertion: Complexities of the surgical intervention include soft-tissue interaction, lesion targeting, and needle flexion. Manual needle insertion is achieved using rigid needles with an assumption of nearly straight insertion; however, this usually results in a lesion shift due to tissue deformation [94]. Researchers focus on thinner and flexible needles to reduce tissue damage and edema with steerability improvements in recent years. The steerability of flexible bevel-tip needle modeling is performed based on duty-cycle rotation models in 2D and 3D during insertion; it allows proportional control of the curvature. These models need real-time imaging feedback for needle tip tracking, which is challenging under MR-imaging due to imaging update times. Moreira et al. [94] presented the first MRI-guided needle tiptracking in phantom using a flexible needle steering algorithm under MRI feedback. They reported targeting errors of 4.3 $\mathrm{mm}, 28 \%$ lower as compared to rigid needles. Performing manual tracking of target and needle, with manual insertion and rotation, and dividing insertion steps to $1.5 \mathrm{~mm}$ each, it takes around 7 minutes to perform each step. Automation of such needles requires robust testing for clinical validation to perform a soft-tissue intervention.
Under US imaging, Abayazid et al. [95] presented teleoperated needle steering in 3D, keeping insertion velocity constant, providing visual and vibratory feedback, and only needle rotation was controlled by the user. As a fully automated needle insertion module is critical, a more realistic environment for proof validation and testing is needed. The following paragraph discusses the role of automation levels in understanding the relation of robotized needle insertion.

3) Manual versus automatic prostate treatments: It is important to discuss the different automation (robot-assisted) prostate needle intervention levels compared to manual. The developed concepts and robotic systems for needle steering can be divided into different automation levels mentioned below [86]:

- Level 0: fully manual insertion.

- Level 1: Manual assisted steering (Steered with haptics);

- Level 2: Semi-automated steering (Physician-in-loop);

- Level 3: Fully automated steering.

There is no practice currently reported in the literature in clinical use involve automated steering. Instead, steering is performed manually by the physician. Physician in the loop guarantees the procedural safety of the patient treatment. In the manual insertion, the physician applies forces on TP skin at the needle entry point using finger pressure. These lateral forces applied with the finger enact the needle deflection, making the needle's lateral displacement [96]. Moreover, lateral actuation and axial rotation [97] have their own advantages to the trajectory and targeting.

In current clinical brachytherapy, two steering actions occur, intermittent axial rotation and lateral actuation. It would be possible that the needle's axial rotation and lateral actuation are performed automatically by a robotic assistance system. Simultaneously, the needle is inserted fully manually by the physician, such that the physician is in charge of the most safety-critical tasks during the needle insertion procedure [96]. Rossa et al. [98] presented a semi-automated hand-held system for manual BT needle insertion with autonomous needle rotation to achieve accuracy.

In recent developments, to achieve image-guidance-based closed-loop control, Wartenberg et al. [99] presented active compensation of deviation from initial straight-line trajectory with the rotation of needle with an asymmetric tip to achieve better accuracy with hands-on co-operative control insertion by the physician. The sensation based on insertion force and along with the needle force, acts as continuous feedback to control insertion velocity. Tests were performed in PVC tissue phantoms with stationary targets with the aid of two cameras (as proxy imaging setup) using Gaussian based model applying Continuous Rotation and Variable. Performing three different evaluations, resulting in 1) $9.30 \mathrm{~mm}$ Error $_{\max }$ without active compensation, 2) RMS $=3.79 \mathrm{~mm}$ for autonomous insertion with active-compensation, and 3) RMS $=3.56 \mathrm{~mm}$ for the hands-on user with co-operative insertion.

\section{Prostate Automatic Tracking}

To achieve automatic needle insertion it is essential to track the target organ, to accomplish the task of adaptive dosimetry 
it requires to track the prostate under real-time during intraoperative needle intervention. In robotized BT system, Hungr et al. [100] successfully demonstrated the concept of prostate motion tracking with 3D-TRUS on cadaver.

Prostate tracking under MRI, Tadayyon et al. [101] proposed a multislice-to-volume registration algorithms for intraoperative prostate motion tracking under MRI, their deformable vs rigid algorithm resulting in average registration error of $2.1 \mathrm{~mm}$ and $2.6 \mathrm{~mm}$, respectively. Authors also anticipated their rigid algorithm to account the deformations due to edema [102]. In other work by Xu et al. [103] presented that MRI slice-to-volume registration is sufficient to produce similar outcomes of volume-to-volume registration for intraoperative prostate motion tracking. A survey by Ferrante and Paragois [104] presents comparison of different works for the slice to volume registration of medical images. The integration of registration algorithm with the TPS of MRIguidance robot's will enhance the procedural accuracy of needle placement and seed-placement/dose delivery.

\section{E. MRI based robotic developments for Prostate interventions}

This sub-section reviews the robotized approaches for prostate intervention. We have comprised a comparison of MR-robotic prototypes performing seed delivery or stated working for possible extensions towards LDR-BT or biopsy robots for needle positioning, summarized it in two tables. Table II constitutes the diagnosis and treatment concept with the robot design conception and control, whereas Table II presents information regarding needle (design and control), imaging, and dosimetry.

The first robotized concept of MRI-guided phantom-based intra-operative prostate intervention was reported in 2000 [105] and demonstrated by DiMaio et al. [17] for biopsy under 0.5 T open MRI at Brigham and Women's Hospital, Harvard (BWH) with the aid of $1.5 \mathrm{~T}$ pre-operative images. USM actuated 5 Degrees of Freedom (DOF) serial robot used for $18 \mathrm{G}$ Ti needle placement. Performed needle insertion manually via TP patient positioned in lithotomy by a clinician with an average needle tip accuracy of $1.2 \mathrm{~mm}$. Structure made up of titanium alloy with linear guides of stainless-steel (YHD50). Safety and sterility were addressed in relation to the MR-robot, taking an example of the BT procedure; however, LDR-BT seed delivery was not reported.

Zangos et al. [106] reported a biopsy via transgluteal access on 20 subjects using theInnoMotion robot (CE mark commercialized by InnoMedic GmbH, currently not existing) pneumatically actuated under $1.5 \mathrm{~T}$. Needle tip (NT) median deviation reported $0.9 \mathrm{~mm}(0.3-1.6 \mathrm{~mm})$ using MRI-compatible needle cannula of $15 \mathrm{G}$ and $16 \mathrm{G}$ needle to collect a tissue sample, $15 \mathrm{G}$ was inserted out-bore by robot and verification of target position with T2-true fast overlaying on pre-planned scans under MRI to collect tissue manually with $16 \mathrm{G}$ needle. The reported needle artifact size in $\mathrm{T} 1$ and $\mathrm{T} 2$ imaging sequence is $16 \mathrm{~mm}$ and $9 \mathrm{~mm}$, respectively. For position reference, gadolinium-based markers were used, taking an average set-up time of 2 minutes at MRI and median procedural time of 39 minutes. In general cases, the TG access is not adopted and not convenient due to prostate depth compared to TR or TP; such a concept can be feasible for a specific patient, as mentioned in III-A3 Also, TG is challenging under closed-bore MRI, and LDR-BT via TG has not been reported or studied.

Krieger et al. [21] developed an Access to Prostate Tissue (APT) 2 DOF manipulator operating inside the $1.5 \mathrm{~T} \mathrm{MRI}$, actuated remotely with the manual rotation of flex-shafts. The team claimed the first successful device used a combination of MRI and tracking coils with a needle through TR-access. Gold markers were implanted in 5 patients with an average displacement error of $4.8 \mathrm{~mm}$. Needle guided is used to target position, $18 \mathrm{G}$ Nitinol tube was inserted manually via a curved guide with an average NT accuracy of $1.3 \mathrm{~mm}$. The fiducial markers help in tracking the tool and target position along the needle path. APT-II [38] modification of APT-I to achieve the 3 in 1 objective to perform MR-guided BX, markers, and seeds for LDR-BT via TR. The APT-II received non-significant risk determination from the FDA and was tested with $1.5 \mathrm{~T}$ and $3 \mathrm{~T}$ on 21 patients. APT-II placed gold markers with an accuracy of $1.1 \mathrm{~mm}$. APT-III [55] MR-conditional robot upgraded with PZ (Nanomotion) actuation reported average NT accuracy of 2.4 $\mathrm{mm}$ for BX. Patient to be laid in prone position and robot can be adapted to other positions. The patient was required to move out for needle insertion. In [122] design of APT-IV (3 DOF) was proposed with $\mathrm{PZ}$ (PiezoLEGS ${ }^{\circledR}$ ) using electro-optical encoders with $44 \%$ reduced in length and $23 \%$ in diameter in comparison to APT-III, application not reported. APT-I \& II relied on manual scale sensing with manual marker deposition, though it showed the clinical application scope for LDR-BT. Whereas APT-III adopted an optical encoder for sensing, but LDR-BT/MD was not performed, reported only BX.

Patriciu et al. [26] presented a fully automated 5 DOF PN using pneustep [64] modular MR-safe robotized seed injector for TP-access LDR-BT under $3 \mathrm{~T}$ MRI tested up to 7T [36]. Seeds were kept in funnel-shaped reservoirs and navigated with pneumatic pressure via a tube to the robot. Ceramic seed placement mean accuracy in the phantom was reported 1.14 $\mathrm{mm}$ and in-vivo (canine) dummy BT seed placement median error $2.50 \mathrm{~mm}(1.45-10.54 \mathrm{~mm})$ with median NT error of 2.02 $\mathrm{mm}(0.86-3.18 \mathrm{~mm})$ [22]. In another study, the team reported the mean accuracy of seed and NT positioning as $3.32 \mathrm{~mm}$ and $0.93 \mathrm{~mm}$ [119]. The team used the OncentraBrachy ${ }^{\mathrm{TM}}$ treatment planning system (TPS); it was also used for robot registration with image, verification of targets, dosimetric plan (along with needle and seeds), and providing coordinates of the target [123].

Patient to be required in LLD position for automatic straight or oblique needle (18G Ti) insertion operating intraoperatively without moving out. Ball et al. [65] used Mr-Bot for a biopsy on 5 subjects targeting 30 sites resulting in $2.55 \mathrm{~mm}$ targeting accuracy with a fully-automated biopsy module. No trajectory corrections were desired during operation. On the other hand, such a device's development is exorbitant, and no other canine or patient study for MD or LDR-BT was reported,though performed clinical BX experiment (got FDA clearance [41]). Moreover, the preoperative scans are usually taken in LT, making it a bit complicated for image registrations from preoperative imaging to real-time dosimetry planning for LLD. 


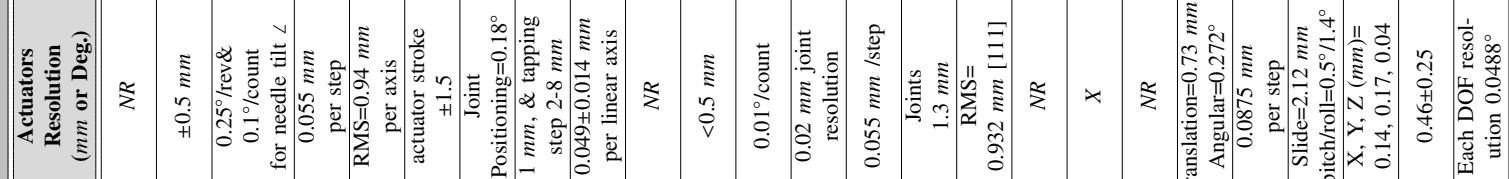

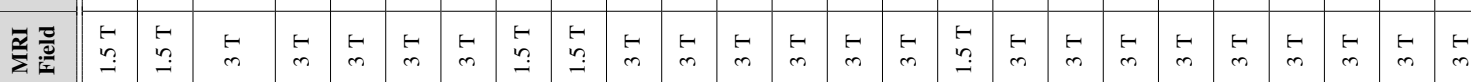

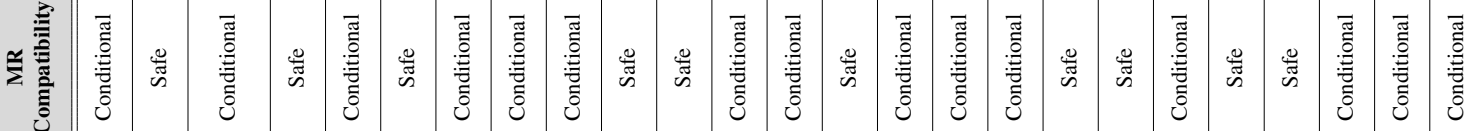

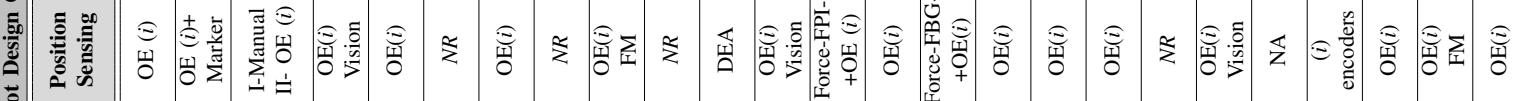

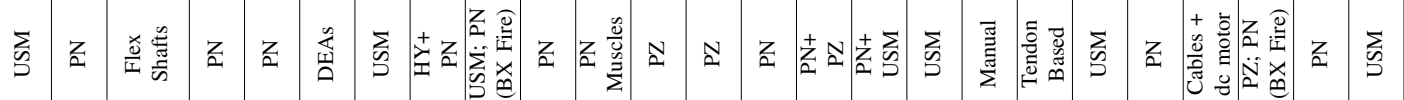

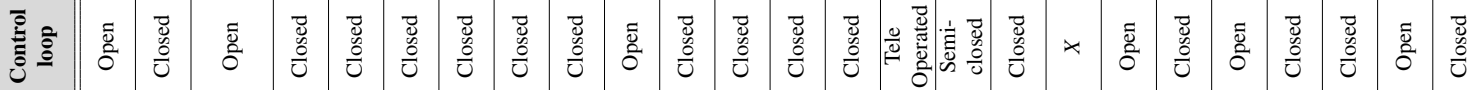

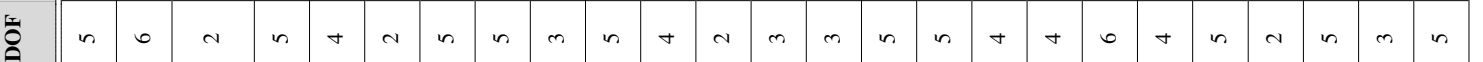

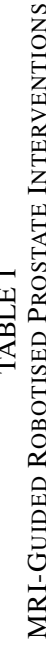

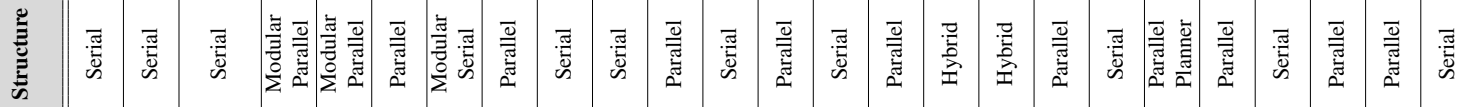

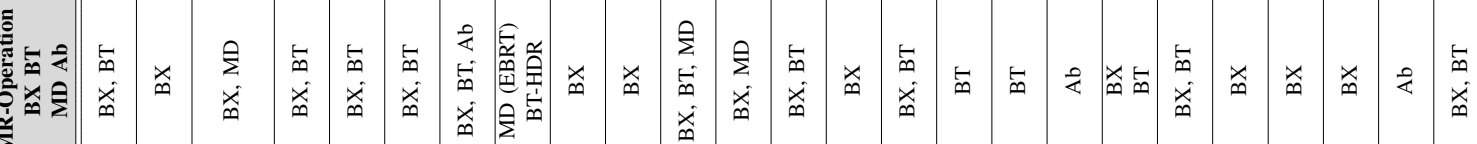

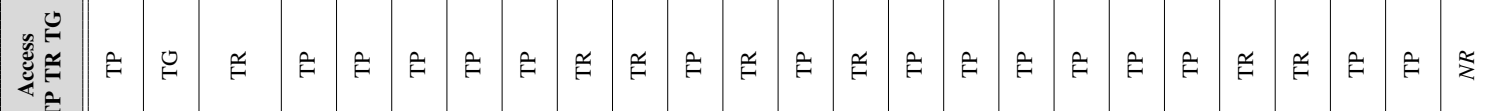

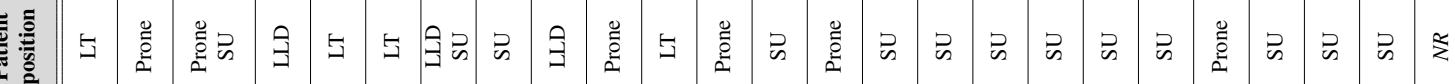

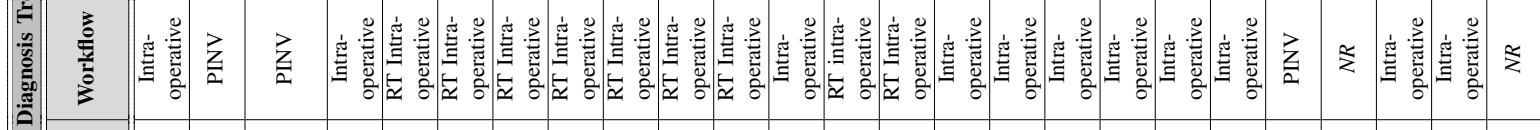

咅

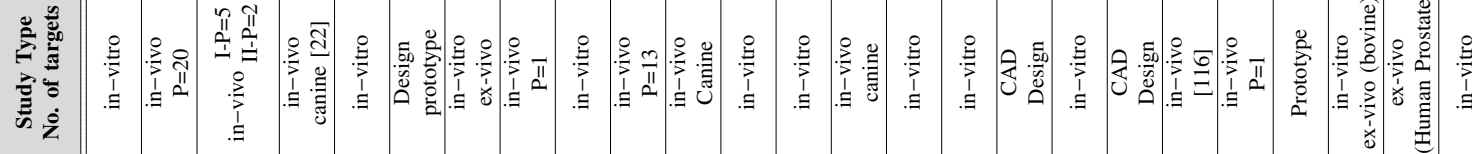

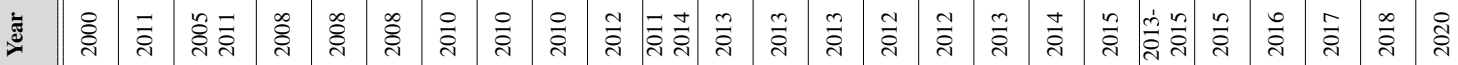

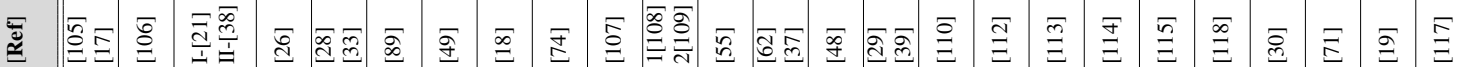

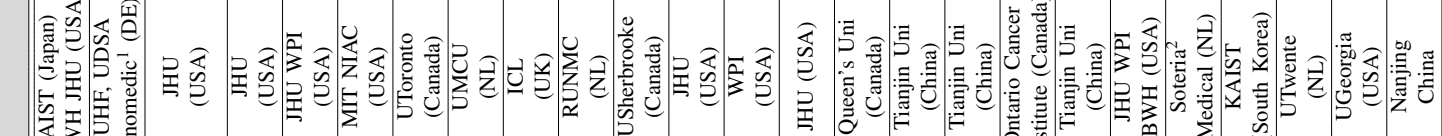

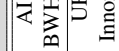




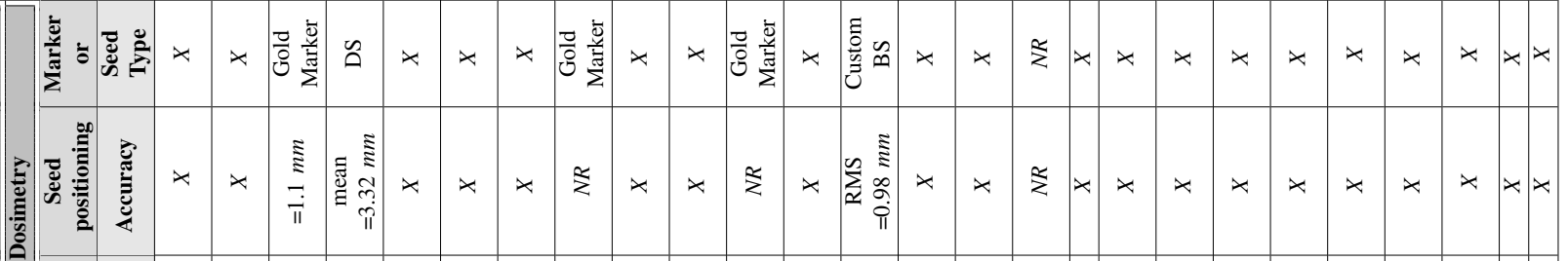

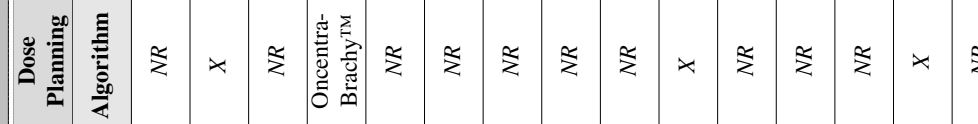

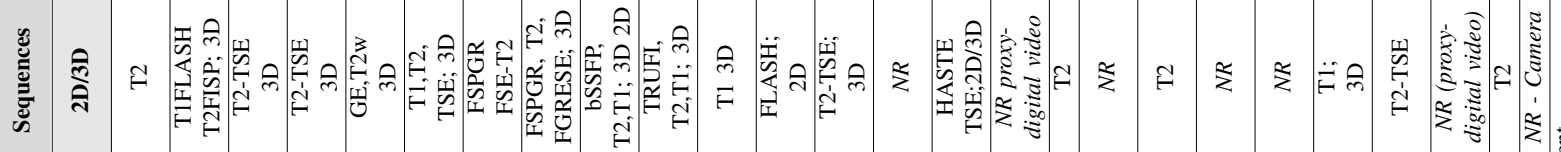

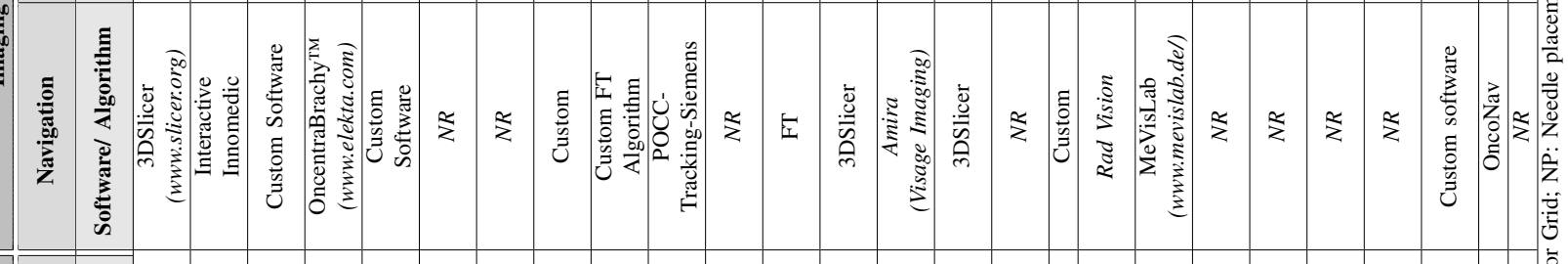

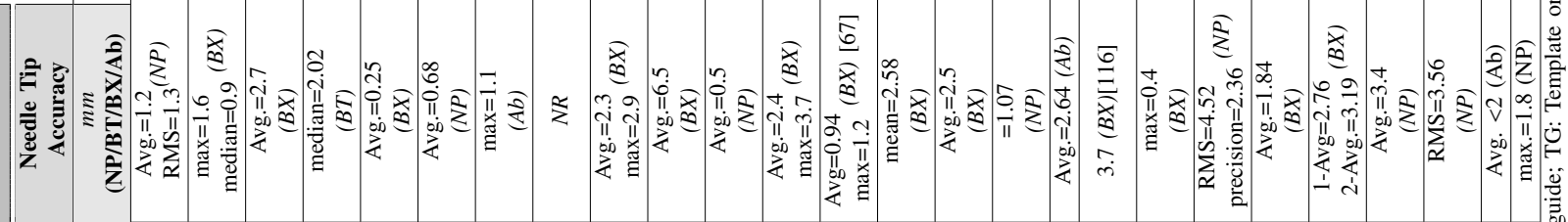

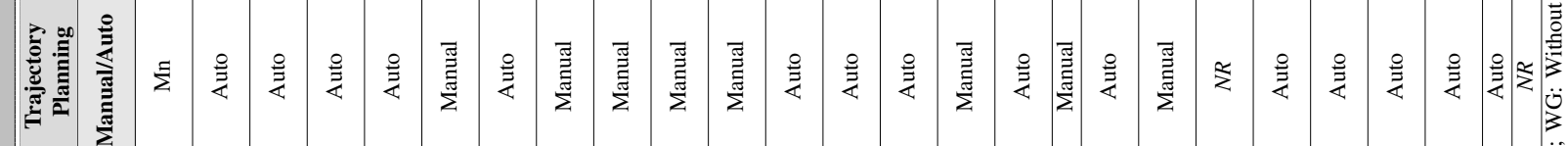

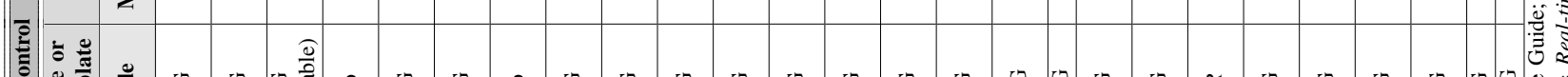

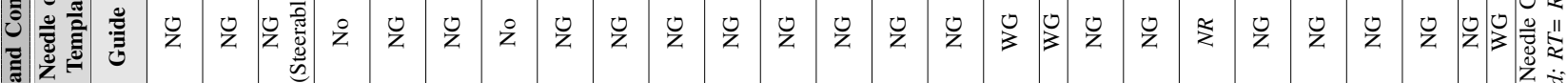

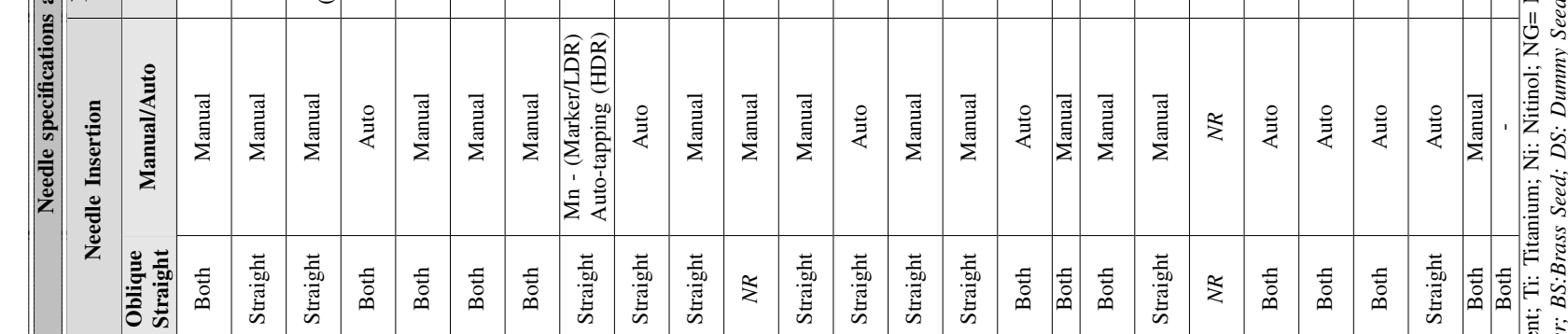

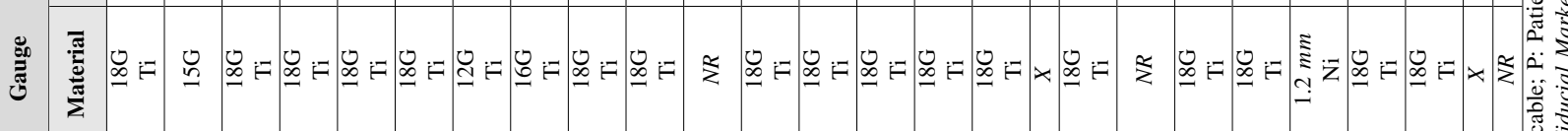

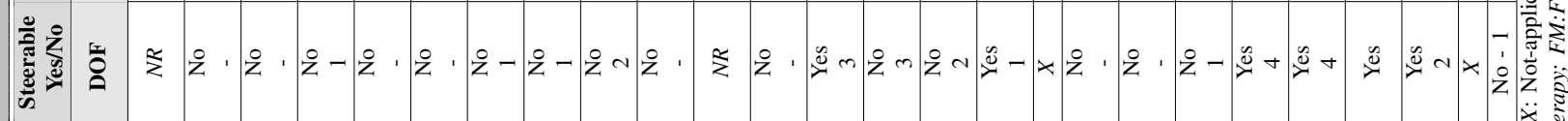

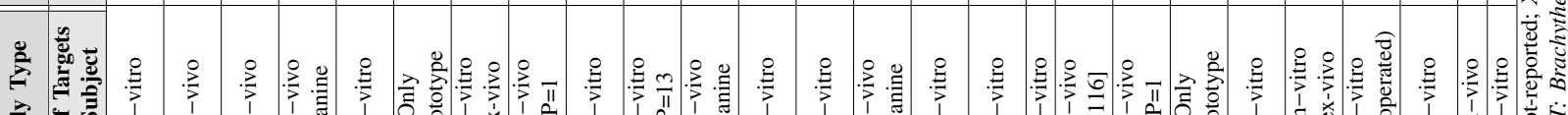

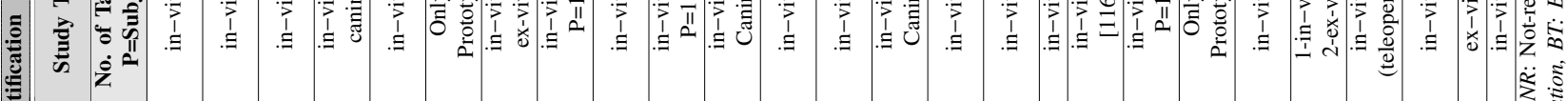

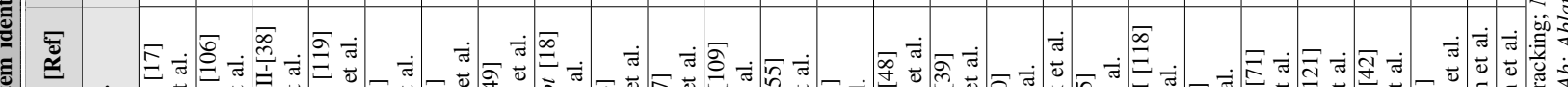

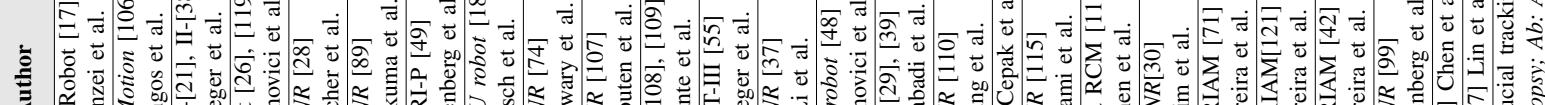

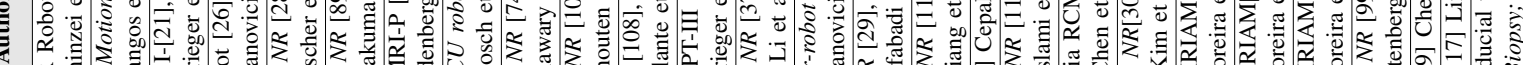

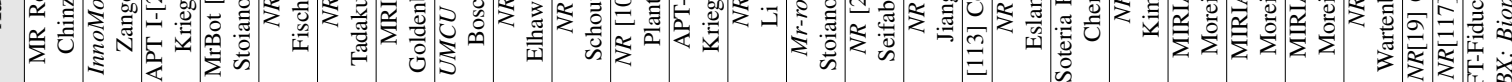


Fischer et al. [28], [33] developed a 4 DOF MRI-conditional PN modular parallel robot mounted upon manual linear slider for needle positioning TP-access in semi-LT under $3 \mathrm{~T}$ MRI. Based on intra-operative image guidance using real-time multiparametric imaging without moving the patient out. The reported average SNR loss was restricted to $5 \%$ of the entire system in the operational mode, with a single axis positioning $R M S$ error of $0.94 \mathrm{~mm}$. NT accuracy $0.25 \mathrm{~mm}$, and the patient moved out for manual needle insertion. 3D-slicer used as navigation software. This work studied architectural aspects of the robotic requirements targeting real-time imaging. However, it required to add needle insertion and rotation, cannula retraction making it 6 DOF. It lacked with clinical study remain limited to in-vitro $\mathrm{BX}$ with manual insertion.

Tadakuma et al. [89] built a high-precision 2 DOF MRSafe parallel manipulator prototype using bistable dielectric elastomer actuation (DEA) experimentally evaluated within a 3 T MRI. The average distance result between experimental and simulated points was about $3 \mathrm{~mm}$ actuation RMS accuracy of $1.4 \mathrm{~mm}$ with $0.5^{\circ}$ using laser calibration. Robot concept with $18 \mathrm{G}$ needle placement straight or oblique via TP approach, maintaining the patient inside the MRI. The issues with this system were a lack of force density and practical durability. During actuation, it is stated 29\% SNR drop and 1\% with power on without actuation. So, DEA may have compatibility and cost advantages, but it lacks to generate the desired forces with reliability for prostate access insertion. It lacked any practical demonstration of needle insertion, presented analytical data.

Goldenberg et al. [49] developed an MRI-P robot with 5 DOF USM actuated via TP, intra-operatively under 1.5 $\mathrm{T}$ MRI without moving the patient out-bore. The physician manipulates the target position and orientation manually with the joystick's help taking feedback from MRI and software only (no force feedback). It was designed for both SU and LLD positions placing the needle straight or oblique. The concept's novelty was a modular structure providing a base to mount a surgical tool to perform ablation, biopsy, and BT. Tests were performed on watermelon and gel phantoms. The MR-Conditional robot resulted in a maximum NT positioning error of $1.1 \mathrm{~mm}$ with a $7 \%$ drop in SNR using a $12 \mathrm{G}$ Nitinol needle. The team demonstrated a robot for radio-frequency ablation only; it lacked a clinical example of BX or BT. It showed that NT accuracy is quite promising with USM.

Bosch et al. [18] demonstrated the first real patient trial with intra-operative robotized MRI-guided gold marker placement in the prostate for EBRT. Hydraulic actuated 5 DOF parallel robot having PN actuated 1 DOF automatic needle drive for straight insertion. Having control accuracy of $<1 \mathrm{~mm}$ and tapping step size between 2 to $8 \mathrm{~mm}$. Patient positioned in LT and gold marker deposited manually using $16 \mathrm{G}$ Ti needle via TP under $1.5 \mathrm{~T}$. Online needle tracking during insertion was performed with fast 2D-images. A robot was made of polymers and non-ferromagnetic materials. It was redesigned using PZ motors and PN needle tapping for HDR. The team stated that in future work to develop navigation software for angular insertion with automatic needle tip tracking against the target. The study of the needle insertion technique is an informative one, however, no additional work was reported for BX or LDR-BT/MD. The robot was limited to needle placement only with manual needle insertion and physician guidance. Parameters like NT and MD accuracy were not reported. The team also presented the concept of adaptive HDR-BT needle insertion sequencing with provisions to integrate their MRrobot, and needle tracking with the aid of FBG sensors under an MRI environment [124].

Elhawary et al. [74] presented a 3 DOF PZ actuated robotguide with a 2 DOF needle module for LLD patient position performing BX via TR inside the MR-bore. It intends to target $\geq 1 \mathrm{~cm}$ lesions with a minimum accuracy of $5 \mathrm{~mm}$, keeping the doctor in the loop, not fully autonomous due to safety measures. The doctor controls the robot based upon realtime MRI imaging. Custom endorectal probe embedded with radio-frequency coil and passive markers having biopsy needle channel was used, made of Duraform material for sterilization. Embedded markers help in real-time guidance. Piezoresistive force sensors were used, the custom pneumatic cylinder with 1.5 bar was designed to fire biopsy needle and harness tissue. Work presented real-time intra-operative; however, it remains limited to in-vitro BX experiments.

Song et al. [29] developed a 4 DOF PN parallel robot, targeting prostate via $\mathrm{TP}$ in $\mathrm{SU}$ position using custom-made leg-rest, with manual $18 \mathrm{G}$ Ti needle insertion under $1.5 / 3 \mathrm{~T}$. Adapted Fischer et al.'s [33] concept modified the Airpel 9.3 bore cylinder with damping for actuation with needle positioning $0.8 \mathrm{~mm}$ [125] 15\% SNR reduction [29]. It can place needle straight and oblique with NT average error of $5 \mathrm{~mm}$. Seifabadi et al. [39] adopted it to fully-automated for intra-operative via teleoperate control, adding PZ actuators to biopsy needle module with average NT accuracy of $2.5 \mathrm{~mm}$ with positioning error $<1 \mathrm{~mm}$ for straight manual needle insertion. Fiber Bragg grating (FBG) sensors opted for force feedback. This MRConditional robot uses a 3D slicer as navigation software. The pyramidal design utilizes space beneath the legs making it workspace efficient. This work with an addition of force sensor and teleoperation achieved needle positioning $<1 \mathrm{~mm}$ with manual insertion; clinical work not reported. However, haptic feedback was not available.

Su et al. [62] developed a modular haptic system with 6 DOF (3 DoF for Cartesian and 3 DOF for needle driver), stating it to be the first PZ actuated prototype to perform needle insertion under Interactive MRI guidance with $3 \mathrm{~T}$ MRI for prostate BT. The prototype works for both biopsy and BT needles in semi-LT position via TP. The team developed its own force sensors to measure in-vivo needle insertion forces (axially, $1 \mathrm{DOF}$ ) for the BT procedure, using FPI sensors with optical encoders from US digital. No significant signal degradation with a $95 \%$ confidence interval. SNR loss is limited to $2 \%$ while imaging and NT placement RMS error of 0.61-2.24 $\mathrm{mm}$ for 3 trajectories. Later gelatin phantom experiments for brachytherapy with custom brass seed (CBS) placement showed an accuracy with RMS error $0.98 \mathrm{~mm}$ targeting $9 \mathrm{~mm}$ bean with automatic straight $18 \mathrm{G}$ steerable needle insertion [37]. Ji et al. [126] extended it to 4 DOF for needle driver with a cylindrical helix imaging coordinate registration fiducial frame for intraoperative intervention. Using the 
same robot guide, Patel et al. [127] demonstrate asymmetricsteerable needle with targeting error $2.5 \mathrm{~mm}$ in gelatin (homogeneous) phantom tracked in $10 \mathrm{~mm}$ slice thickness using fully automatic as closed-loop steering. Still, its feasibility with biological tissue is challenging. The same robot in [67] mounted with needle module showed single-joint motion accuracy of $0.03 \mathrm{~mm}$ and biopsy NT accuracy of $0.87 \mathrm{~mm} \pm$ $0.24 \mathrm{~mm}$. The team extended it to convert it into a telesurgery system adopting PN haptic system + force sensing (straingauge) for needle steering (rotation + insertion) with in-bore demonstration, physician getting haptic and visual (real-time intra-operative) feedback to control insertion [77]. In [37], the work is informative in different aspects, demonstrating CBS's sub-millimeter accuracy. However, TPS regarding dosimetry planning and the clinical application was not discussed.

Schouten et al. [107] performed testing of PN actuated 5 DOF on 13 patients controlling the robot in open-loop and reported a mean biopsy error of $6.5 \mathrm{~mm}$ and compared the procedural time between manual and robotized biopsy. The robotized BX took more procedural time as compared to manual; still, it demonstrated robotic-BX feasibility. The work showed a mean target displacement of $6.6 \mathrm{~mm}$. In [128], the same system studied the clinical feasibility of tracking algorithmbased sequence phase-only cross-correlation (POCC) for realtime MRI biopsy, a procedure targeting automatic tracking of the needle guide.

Jiang et al. [110] developed a 5 DOF prototype with PN actuation and USM for needle insertion with a control accuracy of $0.9 \mathrm{~mm}$ under the digital video. NT error of 1.07 $\mathrm{mm}$. The robot is designed to operate under a $1.5 \mathrm{~T} \mathrm{MR}$ scanner for SU position via TP. In the other two robotized concepts for prostate intervention, Jiang et al. [112] discuss compact USM actuated virtual prototype design of 4 DOF robot with 1 DOF needle insertion. This MRI-conditional robot desire patient to in an SU position with automatic insertion via TP. Jiang et al. [114] presented another virtual prototype of an MR-Safe tendon-based 5 DOF robot +1 DOF needle insertion module for intra-operative BX and BT. Patient to be in an SU position for automatic needle insertion under 0.5 to 3 T MRI scanner. However, no in-vitro or clinical application demonstration was reported for LDR-BT. The team stated in [129] that trajectory planning uses a 3D dynamic algorithm for an $18 \mathrm{G}$ Ti needle.

Plante et al. [108] introduced the embedded air-muscles without any mechanical joints replacing DEAs used in similar design [89]. The targeted insertion force of $0.32 \mathrm{~N} / \mathrm{mm}$ without experiencing trajectory deflections assumes prostate depth around 60-160 $\mathrm{mm}$ via perineum, using 20 air-muscles for $3 \mathrm{~T}$ MRI. The air-muscles are with a radially reinforced membrane with molded ribs limiting the radial expansion and benefiting the extension due to pressure. The team increased air-muscles from 12 to 20 and observed an increase in the numbers, and they were able to drop the hysteresis errors; it also reduced overall size in diameter. Work demonstrated the clinical feasibility of binary muscles for MR-robotized concept to deposit fiducial markers in [109]. However, the needle needs to be guided manually with a fiber-glass needle-guide; needletip and marker-deposition accuracy were not reported.
Eslami et al. [115] presented a parallel kinematic robot with 4 DOF USM actuated aid in target alignment to needle guide for manual needle insertion. Patient positioned in SU/semi-LT with custom leg-support and designed for $3 \mathrm{~T}$ MRI. MRIConditional robot $18 \mathrm{G} \mathrm{Ti}$, NT accuracy of $1 \mathrm{~mm}$ in the air with control accuracy of $0.73 \mathrm{~mm}$ for translation and $0.272^{\circ}$ for orientation. It intends to help clinicians perform manual needle insertion in oblique or straight via TP for intra-operative BX and BT. The SNR was relatively stable, with a variation of no more than $15.35 \%$. The navigation software to be used is RadVision ${ }^{\mathrm{TM}}$. However, it lacked clinical application or in-vitro testing and was limited to manual needle insertion. Wartenberg et al. [99] extended the concept of co-operative control with active compensation of needle deflection to keep the physician in the loop to achieve intra-operatively closedloop control and demonstrated RMS accuracy of $3.56 \mathrm{~mm}$.

Chen et al. [118] presented an MR-Safe PN robot of compact design with $5 \mathrm{DOF}$, commercialized by Soteria Medical for prostate $\mathrm{BX}$ while keeping the patient in a prone position. The robot aids in needle guide positioning and the physician performs manual needle insertion with semiautomated guidance under manual trajectory planning for navigation using MeVisLab software. The authors reported NT placement clinically with a mean accuracy of $0.6 \mathrm{~mm}$ and $2.5^{\circ}$. A clinical study on 57 subjects was conducted and reported reduced procedural time as compared to manual MRI-guided BX and cost-effective with reduced occupancy of MRI while using RCM-robot, however, NT placement accuracy was not reported [130].

Kim et al. [30] proposed a cable-driven 3 Dof (1 needle insertion) MR-Safe robot for prostate biopsy, patient in a supine position. The manipulator controlled using the Maxon $^{\circledR}$ dc-motors using pull-pull Dyneema ${ }^{\circledR}$ cables with cable-pulley transmission kept outside the scanner room. The time delay control (TDC) approach was used for needle positioning resulting in RMS error $=4.52 \mathrm{~mm}$ and $\max$ error $=2.36$ $\mathrm{mm}$. The authors applied a backlash compensator and reported about a $50 \%$ reduction in $\max$ error $=1.86 \mathrm{~mm}[131]$.

Misra et al. [71] presented 5 DOF parallel robot architecture with PZ motor and a 4 DOF needle driver with PZ (Nanomotion) actuated needle (flexible bevel-tip) for insertion and pneumatic firing to harvest tissue sample with a clinically approved BX-needle. The system can perform a fully automated BX and demonstrate multiple steps cross-checking the needle position each time with MRI. The robot helps in positioning and orienting the needle-guide along with TP. The team stated that their contribution is the first one with an integrated robot, pre-operative plan, needle tip (flexible bevel-tip) tracking with steering control to compensate for the deviations that occurred due to tissue's deflections and deformation. Positioning the patient in semi-LT, insertion via TP used commercial (Sterylab) 18G BX-needle. Using optical rotary encoders for measurement and fiducial-markers opted to locate the robot in the MRI coordinate frame. Reflective optical encoder opted for needle depth insertion. The team used random minimization and random path generator algorithms for automated trajectory planning generation.

In addition, the team presented in [121] the fusion of MR 
imaging with flexible needle steering using FBG sensor-based tracking in a closed-loop within MRI bore. Custom designed flexible Nitinol biopsy needle to collect tissues demonstrated replacing solid-Nitinol bevel-tip wires [127] and having 2.5 times larger curvature than a commercial biopsy needle. The needle deflection model is presented in [132], where fiducial markers were used for robot registration. The team used an Acoustic Radiation Force Impulse technique to estimate and calculate the stiffness of soft-tissue. This information helps in haptic control for needle insertion to overcome force sensor placement issues within the MR scanner.

Lin et al. [117] presented a 6 DOF serial mechanism robot for prostate intervention, actuated using 6 USMs (3 for Cartesian motion, 2 for needle orientation, and 1 for needle insertion). Authors measured $1.89 \mathrm{~mm}$ needle-tip accuracy in in-vitro tests using binocular digital cameras. The motors driving controllers were customized. The robot is MRConditional and with the compact design, it is suitable for in-bore placement along with patients, however, the patient position is not stated as development is in progress.

\section{F. Automated LDR-BT Seed Delivery Device}

To develop a fully-automated robotized system for seed delivery it is essential to equip the robot-guide with a seed loading device, not limiting the robot-guide to needle placement only to the target organ.

In our survey, the only commercialized automated seeddelivery system to date is SeedSelectron ${ }^{\circledR}$ by Nucletron (Netherlands). Other automated systems developed are by 1) Patriciu et al. [26] seed-injector with MrBot (MRI-Safe robot), 2) Merzouki et al. [135] developed fully-automated seed loader device and needle placement robot for TRUSBT, the team is currently adapting this concept under MRIenvironment in the frame work of on-going CoBra project with the participation of Eckert \& Ziegler BEBIG GmbH, 3) Sato et al. [136] developed a remote seed-loader device pneumatic actuated using air-pressure to migrate seed from the reservoir to needle, 4) Proffitt et al. [137] developed a non-mechanical design actuated using pneumatic actuation. Concerning the commercialized passive systems for seedloading are such as Mick ${ }^{\circledR}$ TP/TPV Applicator and Isocord systems Eckert \& Ziegler BEBIG GmbH ${ }^{19}$ are available. Taking an account of automated seed delivery device for MRIenvironment, only one such automated seed delivery module is based on pneumatic actuation is developed by Patriciu et al. [26], and integrated to MRI-Safe robot. The device made by Sato et al. [136] and Proffitt et al. [137] can also be possibly extended to MRI-environment due to its pneumatic actuation principle for seed navigation from reservoir to needle and followed by automated mandrel to the delivery site.

\section{IMAGING AND PROCESSING}

It is well known that MRI generates better visualization of soft tissue than alternative clinical imaging systems such as CT. However, localization of seed and needle tips is

\footnotetext{
${ }^{19}$ https://www.bebig.com/home/products/prostate_seed_brachytherapy/
}

challenging due to artifacts and signal voids or geometric distortions (due to actuators or components in the case of MR-robots). Additionally, seed localization under MRI is challenging due to needle traces resulting in negative contrast. Existing protocols at some hospitals use the fusion of CT (for seeds) and MRI (for anatomy); fusion is used for postimplant dosimetry. However, data accuracy is compromised when using such fusion as changes happen in prostate size appear during the shift in imaging modality from MRI to CT.

This motivated the teams to explore using MRI only as an imaging modality for post-implant dosimetry using positive contrast agent markers, e.g., Sirius ${ }^{20}$

There are several commercialized MR+TRUS fusion software, e.g., Koelis ${ }^{\circledR 21}$ MTT ${ }^{22}$ etc., for biopsy and BT software packages, e.g., VariSeed ${ }^{\mathrm{TM}}$ and OncentraBrachy ${ }^{\mathrm{TM}}$. The accuracy desired for a biopsy is not sub-millimeter as compared to the BT implants [133]. The commercial BT planning tools are based on manual point-based, contour-based, or automated mutual information algorithms. The limitation of rigid registration is that it does not account for tissue deformations[133].

Intra-operative image guidance is generally performed at lower resolution because of fast-image acquisition in the presence of a surgical tool [138]. It justifies the use of image registration of preoperative images with real-time images and updated iteratively. However, this requires an efficient imageregistration algorithms.

\section{A. Image Acquisition}

Image acquisition is a critical component of MRI-robotized BT treatment. It demands the pelvis's volumetric scan with a different set of MR imaging sequences (T1-w, T2-w, GRE, SE, etc.) for prostate, OARs, lesion characterization, and imaging-tool for robot guidance. T1-w serve the purpose of prostate definition, and $\mathrm{T} 2-\mathrm{W}$ are used to delineate the substructures, OARs, and determines the lesion sites.

The 3D geometry generated from T2-w images can be correlated with $\mathrm{T} 1-\mathrm{W}$ or real-time sequences for an implant. Also, T2-w images alone are not reliable for needle guidance in the case of the thin peripheral zone[139]. With the aid of registration markers as reference points during imaging, it helps to define the relative target position to track the changes in anatomy during implant.

For diagnostic purposes, the image acquisition sequences have been optimized, but this is not yet the case for BT treatment or seed detection under real-time MRI, which are under research [10]. Imaging protocol optimization is challenging due to the involvement of different parts in BT, i.e., a) needle, b) seeds, c) prostate, d) lesions, e) OARs, and f) markers; each is having its own magnetic susceptibility and influence on imaging. Another reason is that the different vendors have their own standard protocols based on different hardware and software. For imaging protocol, performing realtime BT requires optimized MR-sequences that cater to BT

\footnotetext{
${ }^{26}$ http://www.c4imaging.com/sirius.html

21 https://koelis.com/en/

${ }^{22}$ https://www.medical-tt.com/en/medicine/ldr-brachytherapy/
} 
components' collaborative influence with clear image and lesser artifacts for precise seed deposition.

Initial developments were based on 3D-geometric models, and deformed model consideration for needle insertion is under progress.

\section{B. Needle Detection}

A study carried out by Penzkofer et al. [142] investigated interventional needle artifacts depending upon the type (needle material/mandrin), orientation, and imaging sequences (BSSFP \& T1-SPGR) within porcine tissue under $1.5 \mathrm{~T}$ and $3 \mathrm{~T}$. The authors stated there was no clear-cut co-relation between needle diameter and material, and artifact size. A significant factor noted was needle orientation affecting the artifact and the needle mandrin material instead of the needle itself. Nickel and steel mandrins resulted in larger artifacts. Also, surface coating/processing of needle plays a role in less pronounced artifacts with $\mathrm{Ni}$. A glass fiber mandrin could be selected for small lesion targets to avoid larger artifacts. In the case of $3 \mathrm{~T}$, shorter echo times were chosen to reduce the impact of higher field strength on artifacts. Clinically the differences were insignificant for $1.5 \mathrm{~T}$ and $3 \mathrm{~T}$; though statistically, they were different.

The systems developed for prostate intervention with an approach of MRI intra-operative needle insertion could not get as many benefits from the MR-imaging. Currently, most of the systems perform open-loop insertion and do not utilize image-feedback to compensate for the deviations by adjusting the trajectory during needle insertion. In addition, they lack modeling of the target motion and needle deflection in deformed soft-tissue.

\section{Needle Guidance System}

The needle holder's navigation under direct MRI to perform real-time needle tracking can be categorized into passive and active guidance systems [133].

1) Passive guidance systems are based upon the surgical tool's susceptibility artifacts inside the patient's body or fiducial marker tracking placed along the patient body. This type of tracking requires more time for better image acquisition and induces errors in fiducial markers segmentation. Suppose the refresh rate frequency is increased in order to improve the situation. In that case, there is a subsequent reduction in time output resolution and needle contrast relative to the tissue, which complicates the tracking with respect to surrounding tissues. For example, in passive fiducial tracking MR-spot contrast, fiducials like Z-frame (6 DOF frame posing) [72] are adopted for registration to patient co-ordinate system (RAS = right-anterior-superior). The Z-frame's registration accuracy in [67] stated subpixel resolution in translation with mean error $=0.27 \mathrm{~mm}$ and $0.16^{\circ}$ in orientation. The Z-frame attached to the robot base is detected in an MRI, and locating the needle driver based on the encoder position from kinematics [33]. Thus, Passive guidance systems lack accuracy and are sensitive to artifacts. Considering the work of robotic system other than prostate for in-bore needle intervention with the aid of passive markers under $3 \mathrm{~T}$ MRI for clinical testing, resulted in $<5 \mathrm{~mm}$ accuracy with $\mathrm{T} 1-\mathrm{w}$ sequences, targeting $10 \mathrm{~cm}$ deep lesion sites of $\geq 5 \mathrm{~mm}$ size [141].

2) Active guidance systems require integration of the RF-coil interventional tool, e.g., needle tip, which does not require any space co-ordinate registration. In this way, it can update the scan-plane according to needle tip position. Still, we notice that this lacks information about the actual needle orientation, which is vital for navigation. Active fiducial tracking was demonstrated for prostate intervention in [21] and for gynecologic interstitial BT in [143]. In general, active guidance systems have high accuracy but are complex, demand dedicated hardware such as small MR-compatible sensors [133]; furthermore, it requires specific imaging sequences to process RF coil signals. Additionally, sterilization of integrated coils remains a challenging task [138].

\section{Seed Localization}

Seed localization is a critical component of LDR-BT. Still, there are challenges of seed visualization during intraoperative treatment under MRI, which need specific image sequences for accurate seed placement. Also, seed verification is challenging due to induced artifacts under real-time MRI. Kuo et al. [144] presented an image processing algorithm for prostate BT-seed localization based on Inversion-Recovery with $O N$-resonant water suppression generating seed's positive contrast under MRI.

\section{E. Use of Endorectal coil (ERC) vs. Standard MRI in treat- ment planning}

A study by Albert et al. [140] suggested that the endorectal coil resulted in prostate distortions and a smaller mean measured prostate volume than TRUS. The study also stated that TRUS overestimates the prostate length, whereas standard MRI has imaging advantages in treatment planning and improved dose homogeneity. The rectal obturator was used to help in prostate stabilization. The study also stated accurate and better dose delivery control with MRI, thus resulting in less toxicity. As prostate intervention based on MRI does not require a TRUS probe or an ERC, the issue of prostate deformation and movement induced by such probes is eliminated. Inflatable ERCs potentially cause more prostate distortion as compared to rigid-ERCs [25].

\section{DOSIMETRY}

The process of treatment planning in radiotherapy has evolved tremendously as a function of the complexity of treatment delivery. Before the year 2000 this process was mainly manual. An operator (dosimetrist, or medical physicist) selected a limited $(<5)$ number of beams and optimized the shape (using the multi-leaf collimator, MLC) and the weights of the individual beams to obtain a more or less conformal dose distribution with a homogeneous dose in the target volume. With the introduction of IMRT (Intensity Modulated Radiotherapy), the treatment planning process become that complicated with a large number of degrees of freedom, that this process needed to be semi-automated [145], [146]. Inverse 
treatment planning consists of defining a number of constraints and objectives, using a built-in objective function and an optimization algorithm (e.g. gradient descent). The treatment planning software will then come up with a solution that can then iteratively be fine-tuned by the operator. In principle the process still demanded a manual intervention of the operator. More recently, several solution have been proposed to go towards fully automated treatment planning. Several solution are available already in commercial treatment planning systems [147]. One solution is to mimicking the iterative process of a human operator. Another solution consists of using a large database of treatment plans that serve as a reference for a new plan (by selecting the most similar patient from that database and "copying" the corresponding treatment plan. A third solution is based on generating a so-called pareto-optimal plan (or even a series of such plans, allowing navigation to select the most appropriate plan for the patient: multi-criteria optimization. Recent methods are based on deep learning, which provides very fast automatic optimization [148].

Implementing the above discussed ideas on BT towards a fully automated solution, especially when adapting the plan in real-time using the MRI images. For LDR treatment one can imagine that a geometrical optimization will automatically lead to an optimal dose distribution due to the short range of the low-energy photons and electrons. For HDR, using an isotope (Ir-192) emitting higher energy photons, a more precise dose guided optimization should be performed, as described above. The treatment plan should ensure that the prostate receives a radiation dose high enough to destroy cancer while sparing healthy surrounding tissues and the internal urethral sensitive structure as far as possible. Planning using the MRI images can improve dose planning, improving target coverage, and reduce the dose to OARs [149].

Conventional dosimetric planning of LDR prostate BT implants is based on TRUS imaging. At the start of the procedure, the US of the prostate is acquired, and the prostate and OARs are contoured. Automatic or manual optimization of needle and seed positions is performed based on the anatomical models and pre-defined dose tolerances. As the needles are inserted under live US guidance, their position is updated manually within the software, due to any small inaccuracies in actual placement against the intended dose, with a corresponding update in the planned dose distribution. The user may choose to implant all needles followed by all seeds or implant and deliver the seeds needle by needle. Either way, future needle or seed positions are updated manually on the software based on the final position of the needles and seeds already implanted. Implanted needles and seeds may have deviated from their planned position due to soft tissue and needle interactions, edema, or clarifications. Also, image quality makes it very difficult to assess a particular seed's true end location.

Generally, the dose distribution calculation are based on unbounded homogeneous water phantom calculations according to the TG43 algorithm [150]. The brachytherapy planning system then convolves a total dose distribution from all sources based on the known dose distribution around a single source. This is undertaken irrespective of any heterogeneities within the calculation volume.

Dose-calculation uncertainties are more pronounced with low-energy 125-I and 103-Pd sources for LDR prostate BT because of the radioactive seeds' high-density material and relatively low energy of emissions. In reference [151], several heterogeneity corrections have been introduced for BT, which can be accounted for in model-based dose-calculation methodologies [152].

For dose calculation in radiation therapy, TPS require pixelvalues directly related to the electron density in order to make attenuation calculations, which is not the case under MRI at this stage. That is the reason MRI data requires conversion into pseudo CT or synthetic CT (sCT). In radiotherapy, to achieve MRI-only workflow, an accurate sCT generation is essential. Further, to attain sCT from MRI (T2w-images) requires the network's training from the medical data-sets [153]. Currently, research is ongoing to involve deep-learning in prostate BT with automatic segmentation and reconstruction of implant needle, lesion delineation, and real-time optimization of the treatment planning with dose-calculation, shown promising results for clinical BT [154]. However, deep-learning has not been researched much for dose-calculation; Mao et al. [155] presented a fast deep-learning BT model with more rapid calculation than the Monte Carlo algorithm with similar accuracy.

\section{A. Real-Time Dosimetry under MRI}

Cormack et al. [157] define a clinical method of real-time dosimetry under MRI using transperineal BT, with a template for dose delivery. The software calculates the dosimetry based on inserted needles before delivery of seeds suggesting the implants based on dosimetric feedback as compared to geometric feedback. It is well known that the seeds show displacement during delivery, and dosimetric calculation is based on inserted needles. It is essential to adopt real-time dosimetric calculation and adapt towards the next seed delivery under real-time MRI to target seed deposition with $\leq 2 \mathrm{~mm}$ accuracy.

Real-time dosimetry implant stages under MRI can be described as 1) patient and imaging coil placement, 2) imaging, 3) target segmentation and dosimetry planning, 4) Needle placement with positional feedback adaptation algorithms to track the shifting target, and 5) Seed delivery to the target site. Table II shows the main concepts of existing MRI robotic systems for prostate intervention systems; the dosimetry column indicates work related to dose-planning software, and source positioning. The systems stated for the prostate needle placement are enlisted, and some systems that did not report the dosimetry work are labeled as $(N R)$, and robots studied for needle placement developed without BT's objective indicated as not-applicable $(X)$.

\section{B. Dosimetry and Edema}

Another critical factor is prostate edema needs to be accounted for accurate dose delivery to soft tissues. The causes of prostate edema can vary and depends up the number of needle puncture, time, re-insertion and target adjustment, needle maneuvering to reach the target. Thus, edema influences 
the displacement of seeds post-implant dosimetry. Edema can cause short term disruption to planned positions for seed implants, due to swelling during the procedure which requires re-optimization of planned seed positions, and also that edema can cause differences between assessed dosimetry at the time of implant and that assessed by post-implant CT dosimetry up to 30 days later when edema will have resolved [158]. The topic is out of scope for this paper to fully elaborate and cover it, and predictive models of edema with real-time needle insertions can improve the overall dose planning. Westendorp et al. [159] presented a study comparing the edema and seed displacement in TRUS and CT based dosimetry with day 30, stated that seeds delivered near to the rectum show more displacement possibly due to the US probe. Mountris et al. [160] presented a study to model edema in LDR-BT-based computed deformations and integrating them to Monte Carlo simulation for dose-estimations. The study claimed the model benefit the overall treatment planning in dosimetry predictions and help in post-implant dosimetry.

\section{DISCUSSION}

This review has considered robotized developments for prostate cancer diagnosis and treatment using MRI. MRI's imaging capabilities have revolutionized the quality of anatomical visualization in oncology procedures. To achieve optimal benefits of MRI for prostate brachytherapy and biopsy under robotic interventions, these should be conducted within the MRI bore, under real-time imaging. Conventionally, ultrasound provided the required real-time imaging but has relatively poor image quality for precise interventions. In contrast, MRI restricts conventional robotized applications due to the strong magnetic field and material compatibility challenges. Further, there are challenges from software development for needle tip tracking of MR-Conditional needles and image registration with markers for precise seed delivery. Therefore, an integrated solution to take advantage of real-time imaging and robots for clinical treatments is yet to be seen.

The surgical robot needs to comply with IEC 60601-1 standard and risk management process [2] and prove clinical feasibility. The field of robotized needle insertion with realtime MRI feedback for prostate cancer more exploration and research with feasible solutions. There have been only limited studies published, primarily with validation only on phantom studies.

Another approach utilizing TRUS + MRI image fusion has limitations due to image registration and is unable to gain the benefits feasible with real-time MR-imaging. Such limitations drive further research of concepts with the direct involvement of MRI for intra-operative treatment. The feasibility of manual intra-operative treatment [13] motivates to extend the procedure with robotized treatment to attain better accuracy. Full autonomy of surgical robots for clinical procedures is still restricted by medical regulatory bodies and ethical review boards with preference and priority to physicians only in command, limiting the robot only to assist them for more precision maintaining the final decisions with physicians. The patient is in a dynamic state, with internal motion and structure deformation in response to any intervention. In brachytherapy, the prostate and surrounding soft-tissues undergo displacements during needle insertion, the monitoring of which is a critical factor for accurate needle placement adaptation using real-time image guidance.

For a robot to act full-autonomously, these complexities and uncertainties must be well managed, potentially with the use of fiducial markers or real-time organ tracking. This domain that desires require the robot's full-autonomy. Fiducial markers can be used to help the robot adapt.

The other research topics regarding seed deposition (robotized) and visualization (under real-time MRI) need to be explored. To date, only needle positioning accuracy has been evaluated, and practical clinical procedure challenges such as seed-dragging during single seed deposition need mitigation or avoidance during robotized LDR-BT.

Some challenges can be stated for future developments of robotic solutions but not limited to MRI-guided robots, such as accounting the prediction models for edema, accounting prostate motion, seed dragging during needle retraction, neoplasm issues with single needle use, needle vacuum while retracting the mandrel, and last but not least an automated seed loader device functional in-bore or near to the MRI scanner, in order to reduce the procedure time and accomplishing brachytherapy under real-time intraoperatively. As imaging technologies are evolving, in parallel to MRI a MircoUltrasound imaging is also being explored and resulted in promising outcomes for better detection of cancer [161]. In the future, such propositions can enhance robots' precision and accuracy.

\section{CONCLUSION}

Robotics inclusion for cancer treatment is being explored at a rapid rate in the last two decades. The development and integration of the set of component systems of innovative robotized technologies for prostate treatment under the MRI environment face many challenges to be taken care of due to MR-compatibility restrictions. Various research groups have explored the domain of prostate cancer treatment under MRI using robotic devices to attain better accuracy in tumor targeting with encouraging TRL and to address the research advancements, gaps, and challenges according to their expertise.

We presented comparative work for prostate interventions under MRI treatment concepts developed within the last 15 years, which demonstrate feasibility at different TRL levels. Most of them lie between 3 to 4 TRL. These robotic developments need industrialization and clinical clearances after demonstrating the clinical feasibility. This comparative study allows us to make the positioning for the on-going EU CoBra project, which intends to develop an MR-concept for robotic diagnosis and treatment of localized Prostate cancers, targeting TRL-6. The coming work-plan is the progressive clinical trials on laboratory animals in 2021.

\section{Authors' CONTRIBUtions}

SD and RM conceptualized the study, SD and TC designed the manuscript. SD performed a systematic review and drafted 
the manuscript. TC and RM critically reviewed the technical details. SW, WP, and AP critically reviewed and edited the clinical content. NR reviewed and contributed to the dosimetry section. RM arranged the financial support for this study.

\section{REFERENCES}

[1] A.C. Moreno et al., "MRI Image-Guided Low-Dose Rate Brachytherapy for Prostate Cancer," Handbook of Image-Guided Brachytherapy, pp. R319-344, 2017.

[2] T. K. Podder et al., "AAPM and GEC-ESTRO guidelines for imageguided robotic brachytherapy: report of Task Group 192," Medical physics, vol. 41, no. 10, 2014.

[3] C. Ménard et al., "Role of Prostate MR Imaging in Radiation Oncology," Radiologic clinics of North America, vol. 56, no. 2, pp. 319-325, 2018.

[4] J. Wang et al., "Magnetic resonance imaging basics for the prostate brachytherapist," Brachytherapy, vol. 16, no. 4, pp. 715-727, 2017.

[5] JB. Soyez et al., "Multilevel Agent-Based Modeling of System of Systems," IEEE Systems Journal, vol. 11, pp. 2084-2095, 2017.

[6] N. Tsekos et al., "Magnetic resonance-compatible robotic and mechatronics systems for image-guided interventions and rehabilitation: a review study," Annual Review of Biomedical Engineering, vol. 9, pp. 351-87, 2007.

[7] R. Monfaredi et al., "MRI Robots for Needle-Based Interventions: Systems and Technology," Annals of Biomedical Engineering, vol. 46, pp. 1479-1497, 2018.

[8] A. Liberati et al., "The PRISMA Statement for Reporting Systematic Reviews and Meta-Analyses of Studies That Evaluate Health Care Interventions: Explanation and Elaboration," PLoS Medicine, vol. 6, 2009.

[9] S. Choi et al. "The role of magnetic resonance imaging in the detection of prostate cancer," Journal of Urology, vol. 186 4, pp. 1181-82, 2011.

[10] S. J. Frank et al., "Use of magnetic resonance imaging in low-dose-rate and high-dose-rate prostate brachytherapy from diagnosis to treatment assessment: Defining the knowledge gaps, technical challenges, and barriers to implementation," Brachytherapy, vol. 16 4, pp. 672-78, 2017.

[11] S. Abel et al., "Evaluation of intraoperative magnetic resonance imaging/ultrasound fusion optimization for low-dose-rate prostate brachytherapy," Journal of Contemporary Brachytherapy, vol. 9 4, p. 309, 2017.

[12] K. Tanderup et al.,"Magnetic resonance image guided brachytherapy," Seminars in Radiation Oncology, vol. 24, no. 3, pp. 181,191, 2014-07.

[13] C. Ménard et al.,"MRI-guided HDR prostate brachytherapy in standard 1.5T scanner," International Journal of Radiation Oncology • Biology • Physics, vol. 59, no. 5, pp. 1414-1423, 2004.

[14] P. Blanchardet al., "MRI Simulation for LDR Prostate Brachyhtherapy: Can We Replace Ultrasound with MRI for Treatment Planning? Comparison of Pre-Planning, Day 0 and Day 30 MR Dosimetry," Brachytherapy, vol. 15 , p. S57, 2016.

[15] A. D'Amico et al., "Real-time magnetic resonance image-guided interstitial brachytherapy in the treatment of select patients with clinically localized prostate cancer," International Journal of Radiation Oncology - Biology • Physics, vol. 42, no. 3, pp. 507-515, 1998.

[16] A. V. D'Amico et al., "Transperineal magnetic resonance image guided prostate biopsy," The Journal of urology, vol. 164 2, pp. 385-7, 2000.

[17] S. P. DiMaio et al., "Robot-assisted needle placement in open-MRI: system architecture, integration and validation," Computer Aided Surgery: Official Journal of the International Society for Computer Aided Surgery, vol. 121 , pp. 15-24, 2006.

[18] M. R. Van den Bosch et al., "MRI-guided robotic system for transperineal prostate interventions: proof of principle," Physics in Medicine \& Biology, vol. 55, no. 5, p. N133, 2010.

[19] Y. Chen et al.,"Robotic System for MRI-guided Focal Laser Ablation in the Prostate," IEEE/ASME Transactions on Mechatronics, vol. 22, pp. 107-114, 2016.

[20] D. A. Jaffray et al., "A facility for magnetic resonance-guided radiation therapy," Seminars in Radiation Oncology, vol. 24 3, pp. 193-5, 2014.

[21] A. Krieger et al., "Design of a novel MRI compatible manipulator for image guided prostate intervention," IEEE International Conference on Robotics and Automation, 2004. Proceedings. ICRA'04. 2004, vol. 1. IEEE, 2004, pp. 377-382.

[22] M. Muntener et al., "Transperineal prostate intervention: robot for fully automated MR imaging-system description and proof of principle in a canine model," Radiology, vol. 247, no. 2, pp. 543-549, 2008.

[23] A. H. Goenka et al., "CT-Guided Transgluteal Biopsy for Systematic Random Sampling of the Prostate in Patients Without Rectal Access," American Journal of Roentgenology, vol. 205(3), pp. 578-583, 2015.
[24] S. Zangos et al., "MR-guided transgluteal biopsies with an open lowfield system in patients with clinically suspected prostate cancer: technique and preliminary results," European Radiology, vol. 15, no. 1, pp. 174-182, 2005.

[25] P. C. Mozer et al., "Robotic image-guided needle interventions of the prostate," Reviews in Urology, vol. 11, no. 1, p. 7, 2009.

[26] A. Patriciu et al., "Automatic brachytherapy seed placement under MRI guidance," IEEE Transactions on Biomedical Engineering, vol. 54, no. 8, pp. 1499-1506, 2007

[27] A. A. Goldenberg et al., "Robotic System for Closed-Bore MRI-Guided Prostatic Interventions," IEEE/ASME Transactions On Mechatronics, vol. 13, no. 3, pp. 374-379, 2008.

[28] G. S. Fischer et al., "MRI-Compatible Pneumatic Robot for Transperineal Prostate Needle Placement," IEEE/ASME Transactions on Mechatronics, vol. 13, no. 3, pp. 295-305, 2008.

[29] S.-E. Song et al., "Development of a Pneumatic Robot for MRI-guided Transperineal Prostate Biopsy and Brachytherapy: New Approaches," 2010 IEEE International Conference on Robotics and Automation. IEEE, 2010, pp. 2580-2585.

[30] K.-Y. Kim et al., "Design of an MRI-compatible modularized needle driver for In-bore MRI-guided prostate interventions," 2015 15th International Conference on Control, Automation and Systems (ICCAS). IEEE, 2015, pp. 1520-1525.

[31] S. S. Dhaliwal et al., "Cooperative Brachytherapy for Prostate Cancer Under MRI Guidance," $5^{\text {th }}$ International Conference on Advances in Biomedical Engineering. Tripoli, Lebanon: IEEE, Oct. 2019, pp. 1-4. https://doi.org./10.1109/ICABME47164.2019.8940278

[32] S.-E. Song et al., "Development and Preliminary Evaluation of a Motorized Needle Guide Template for MRI-Guided Targeted Prostate Biopsy," IEEE Transactions on Biomedical Engineering, vol. 60, no. 11, pp. 3019-3027, 2013.

[33] G. S. Fischer et al., "Pneumatically Operated MRI-Compatible Needle Placement Robot for Prostate Interventions," 2008 IEEE International Conference on Robotics and Automation, pp. 2489-2495, 2008.

[34] R. Merzouki et al., "CNRS la lettre innovation," http://www.cnrs.fr/ lettre-innovation/actus.php?numero $=341$

[35] R. Seifabadi et al., "Toward teleoperated needle steering under continuous MRI guidance for prostate percutaneous interventions," International Journal of Medical Robotics and Computer Assisted Surgery : MRCAS, vol. 12 3, pp. 355-69, 2016.

[36] M. Muntener et al., "Magnetic resonance imaging compatible robotic system for fully automated brachytherapy seed placement," Urology, vol. 68, no. 6, pp. 1313-1317, 2006.

[37] G. Li et al., "A Fully Actuated Robotic Assistant for MRI-Guided Prostate Biopsy and Brachytherapy," Medical Imaging 2013: ImageGuided Procedures, Robotic Interventions, and Modeling, vol. 8671. International Society for Optics and Photonics, 2013, p. 867117.

[38] A. Krieger et al., "An MRI-Compatible Robotic System With Hybrid Tracking for MRI-Guided Prostate Intervention," IEEE Transactions on Biomedical Engineering, vol. 58, no. 11, pp. 3049-3060, 2011.

[39] R. Seifabadi et al., "Robotic system for MRI-guided prostate biopsy: feasibility of teleoperated needle insertion and ex vivo phantom study," International Journal of Computer Assisted Radiology and Surgery, vol. 7, pp. 181-190, 2011.

[40] F. A. Jolesz, "Intraoperative Imaging and Image-Guided Therapy," Springer, 2014.

[41] D. S. Stoianovici et al., "MR Safe Robot, FDA Clearance, Safety and Feasibility of Prostate Biopsy Clinical Trial," IEEE/ASME Transactions on Mechatronics, vol. 22, pp. 115-126, 2017.

[42] P. Moreira et al., "Tele-Operated MRI-Guided Needle Insertion for Prostate Interventions," Journal of Medical Robotics Research, vol. 4, no. 01, p. 1842003, 2019.

[43] M. Li et al., "MRI Compatible Robot Systems for Medical Intervention," Advances in Robot Manipulators. IntechOpen, 2010.

[44] E. G. Christoforou and N. V. Tsekos, "Robotic systems for MRIguided interventions," Advances in Medical Physics: 2010, 2014, pp. 73-87. [Online]. Available: https://medicalphysics.org/documents/ 2010AdvancesChapter5.pdf

[45] K. Chinzei, R. Kikinis, and F. A. Jolesz, "MR Compatibility of Mechatronic Devices: Design Criteria," International Conference on Medical Image Computing and Computer-Assisted Intervention. Springer, 1999, pp. $1020-1030$.

[46] L. L. Tsai et al., "A Practical Guide to MR Imaging Safety: What Radiologists Need to Know," Radiographics, vol. 35 6, pp. 1722-37, 2015.

[47] "ASTM International - standards and publications," https://www.astm. org/Standard/standards-and-publications.html 
[48] D. S. Stoianovici et al., "MRI-Safe Robot for Endorectal Prostate Biopsy," IEEE/ASME Transactions on Mechatronics, vol. 19, pp. 12891299, 2013.

[49] A. A. Goldenberg et al., "Robot-assisted MRI-guided prostatic interventions," Robotica, vol. 28, no. 2, p. 215-234, 2010

[50] H. Elhawary et al., "A Modular Approach to MRI-Compatible Robotics," IEEE Engineering in Medicine and Biology Magazine, vol. 27, no. 3, pp. 35-41, 2008.

[51] K. Masamune et al., "Development of an MRI-compatible needle insertion manipulator for stereotactic neurosurgery," Journal of Image Guided Surgery, vol. 1, no. 4, pp. 242-248, 1995.

[52] D. Beyersdorff et al., "MR imaging-guided prostate biopsy with a closed MR unit at 1.5 T: initial results," Radiology, vol. 234 2, pp. 576-81, 2005.

[53] K. Bannan et al.,"Prediction of Force and Image Artifacts Under MR for Metals Used in Medical Devices," IEEE/ASME Transactions on Mechatronics, vol. 18, pp. 954-962, 2013.

[54] M. Hara et al., "Design and compatibility of a high-performance actuation system for fMRI-based neuroscience studies," IEEE/RSJ International Conference on Intelligent Robots and Systems, pp. 2437-42, 2010.

[55] A. Krieger et al., "Development and Evaluation of an Actuated MRICompatible Robotic System for MRI-Guided Prostate Intervention," IEEE/ASME Transactions on Mechatronics,vol. 18, pp. 273-284, 2012.

[56] R. Gassert et al., "MRI/fMRI-compatible robotic system with force feedback for interaction with human motion," IEEE/ASME Transactions on Mechatronics, vol. 11, pp. 216-224, 2006.

[57] R. Gassert et al., "Actuation methods for applications in MR environments" Concepts in Magnetic Resonance Part B: Magnetic Resonance Engineering: An Educational Journal, vol. 29 4, pp. 191-209, 2006.

[58] Y. U. Kim et al.,"Effect of patient position on pain scales during transrectal ultrasound-guided prostate biopsy" Korean Journal of Urology, 2015.

[59] H. SU et al.,"Fiber-Optic Force Sensors for MRI-Guided Interventions and Rehabilitation: A Review" IEEE Sensors Journal, vol. 17, pp. 195263, 2017.

[60] P. Vartholomeos et al., "MRI-powered actuators for robotic interventions," 2011 IEEE/RSJ International Conference on Intelligent Robots and Systems, pp. 4508-15, 2011.

[61] G. S. Fischer et al., "MRI compatibility of robot actuation techniques-a comparative study," International Conference on Medical Image Computing and Computer-Assisted Intervention - MICCAI, pp. 509-517, 2008.

[62] H. Su et al., "Real-time MRI-guided needle placement robot with integrated fiber optic force sensing," 2011 IEEE International Conference on Robotics and Automation, pp. 1583-88, 2011.

[63] B. Yang et al., "Design and Control of a 1-DOF MRI-Compatible Pneumatically Actuated Robot With Long Transmission Lines,"IEEE/ASME Transactions on Mechatronics, vol. 16, pp. 1040-48, 2011.

[64] D. Stoianovici et al., "A New Type of Motor: Pneumatic Step Motor," IEEE/ASME Transactions On Mechatronics, vol. 12 1, pp. 98-106, 2007.

[65] M. W. Ball et al.,"Safety and Feasibility of Direct Magnetic Resonance Imaging-guided Transperineal Prostate Biopsy Using a Novel Magnetic Resonance Imaging-safe Robotic Device," Urology, vol. 109, pp. 21621, 2017.

[66] T. Suzuki et al., "Ultrasonic motor driving method for EMI-free image in MR image-guided surgical robotic system," 2007 IEEE/RSJ International Conference on Intelligent Robots and Systems, pp. 522-527, 2007.

[67] H. Su et al., "Piezoelectrically Actuated Robotic System for MRIGuided Prostate Percutaneous Therapy," IEEE/ASME Transactions on Mechatronics, vol. 20, pp. 1920-1932, 2015.

[68] P. Carvalho et al., "Demonstration and Experimental Validation of Plastic-Encased Resonant Ultrasonic Piezoelectric Actuator for Magnetic Resonance Imaging-Guided Surgical Robots," Journal of engineering and science in medical diagnostics and therapy, vol. 3 1, 2020.

[69] W. Wang et al., "Compatibility of US motors for development of MRIguided surgical robot," 2013 6th International Conference on Biomedical Engineering and Informatics,pp. 388-392, 2013.

[70] P. Shokrollahi, J. M. Drake, and A. A. Goldenberg, "Signal-to-noise ratio evaluation of magnetic resonance images in the presence of an ultrasonic motor,"Biomedical engineering online, vol. 16 1, p. 45, 2017.

[71] P. Moreira et al., "The MIRIAM Robot: A Novel Robotic System for MR-Guided Needle Insertion in the Prostate," Journal of Medical Robotics Research, vol. 2, no. 04, p. 1750006, 2017.

[72] S. P. DiMaio et al., "Dynamic MRI scan plane control for passive tracking of instruments and devices," Medical image computing and computer-assisted intervention : MICCAI ... International Conference on Medical Image Computing and Computer-Assisted Intervention, vol. 10 Pt 2, pp. 50-8, 2007.

[73] M. Borot de Battisti, et al., "Fiber Bragg gratings-based sensing for real-time needle tracking during MR-guided brachytherapy," Medical Physics, vol. 43 10, pp. 5288, 2016.

[74] H. Elhawary, et al., "Robotic system for transrectal biopsy of the prostate: real-time guidance under MRI," IEEE Engineering in Medicine and Biology Magazine, vol. 29, no. 2, pp. 78-86, 2010.

[75] R. Kokes et al., "Towards a teleoperated needle driver robot with haptic feedback for RFA of breast tumors under continuous MRI," Medical image analysis, vol. 13, no. 3, pp. 445-455, 2009.

[76] W. Shang et al., "Teleoperation system with hybrid pneumaticpiezoelectric actuation for MRI-guided needle insertion with haptic feedback," 2013 IEEE/RSJ International Conference on Intelligent Robots and Systems, pp. 4092-4098, 2013.

[77] H. Su et al., "An MRI-Guided Telesurgery System Using a Fabry-Perot Interferometry Force Sensor and a Pneumatic Haptic Device," Annals of Biomedical Engineering, vol. 45, pp. 1917-1928, 2017.

[78] H. Hoshyarmanesh, et al., "Laser Registration and Supervisory Control of neuroArm Robotic Surgical System," World Academy of Science, Engineering and Technology, International Journal of Mechanical, Aerospace, Industrial, Mechatronic and Manufacturing Engineering, vol. 11, pp. 1407-1414, 2017.

[79] N. N. Stone et al., "Prostate gland motion and deformation caused by needle placement during brachytherapy," Brachytherapy, vol. 13 , pp. 154-60, 2002

[80] M. J. Dattoli and K. G. Waller, "A simple method to stabilize the prostate during transperineal prostate brachytherapy," International Journal of Radiation Oncology • Biology • Physics, vol. 38 2, pp. 341-2, 1997.

[81] A. M. Okamura, C. Simone, and M. D. O'Leary, "Force modeling for needle insertion into soft tissue," IEEE Transactions on Biomedical Engineering, vol. 51, pp. 1707-1716, 2004.

[82] T. Podder et al., "Vivo motion and force measurement of surgical needle intervention during prostate brachytherapy," Medical Physics, vol. 33, no. 8, pp. 2915-2922, 2006.

[83] T. Podder et al., "Effects of velocity modulation during surgical needle insertion," IEEE Engineering in Medicine and Biology 27th Annual Conference. IEEE, 2005, pp. 5766-5770.

[84] S. E. Salcudean et al., "A robotic needle guide for prostate brachytherapy," 2008 IEEE International Conference on Robotics and Automation, pp. 2975-2981, 2008.

[85] T. K. Podder et al., "A novel curvilinear approach for prostate seed implantation," Medical Physics, vol. 39, no. 4, pp. 1887-1892, 2012.

[86] C. Rossa and M. Tavakoli, "Issues in closed-loop needle steering," Control Engineering Practice, vol. 62, pp. 55 - 69, 2017.

[87] P. Kulkarni et al., "Review of Robotic Needle Guide Systems for Percutaneous Intervention," Annals of Biomedical Engineering, vol. 47, pp. 2489 - 2513, 2019.

[88] H. Su et al., "Active Needle Steering System for Percutaneous Prostate Intervention in High-field MRI,"in Workshop on Enabling Technologies for Image-Guided Robotic Interventional Procedures, 2010, p. 35.

[89] K. Tadakuma et al., "The experimental study of a precision parallel manipulator with binary actuation: With application to MRI cancer treatment," 2008 IEEE International Conference on Robotics and Automation. IEEE, 2008, pp. 2503-2508.

[90] S. Eslami et al., "Towards clinically optimized MRI-guided surgical manipulator for minimally invasive prostate percutaneous interventions: constructive design," 2013 IEEE International Conference on Robotics and Automation. IEEE, 2013, pp. 1228-1233.

[91] H. Su et al., "A concentric tube continuum robot with piezoelectric actuation for MRI-guided closed-loop targeting," Annals of Biomedical Engineering, vol. 44, no. 10, pp. 2863-2873, 2016.

[92] "InvivoCorp - 3T Fully Automatic Biopsy Gun," https://www. documents.philips.com/assets/Instruction\%20for\%20Use/20200623/ 510d22e9c3cf4b8b96dfabe3012c4758.pdf?feed=ifu_docs_feed

[93] I. Khodarahmi et al., "Needle Heating During Interventional Magnetic Resonance Imaging at 1.5- and 3.0-T Field Strengths," Investigative Radiology, vol. 55, pp. 396 - 404, 2020.

[94] P. Moreira et al., "A preliminary evaluation of a flexible needle steering algorithm using magnetic resonance images as feedback," 5th IEEE RAS/EMBS International Conference on Biomedical Robotics and Biomechatronics, pp. 314-319, 2014.

[95] M. Abayazid, N. Shahriari, and S. Misra, "Three-dimensional needle steering towards a localized target in a prostate phantom," 5th IEEE RAS/EMBS International Conference on Biomedical Robotics and Biomechatronics, pp. 7-12, 2014. 
[96] T. Lehmann et al., "Human-Machine Collaboration Modalities for SemiAutomated Needle Insertion Into Soft Tissue," IEEE Robotics and Automation Letters, vol. 3, no. 1, pp. 477-483, 2017.

[97] M. Meltsner, N. Ferrier, and B. Thomadsen, "Observations on rotating needle insertions using a brachytherapy robot," Physics in Medicine \& Biology, vol. 52, no. 19, p. 6027, 2007.

[98] C. Rossa et al., "A Hand-Held Assistant for Semiautomated Percutaneous Needle Steering," IEEE Transactions on Biomedical Engineering, vol. 64, pp. 637-648, 2017.

[99] M. Wartenberg et al., "Closed-Loop Active Compensation for Needle Deflection and Target Shift During Cooperatively Controlled Robotic Needle Insertion," Annals of Biomedical Engineering, vol. 46, pp. 158294, 2018.

[100] N. Hungr et al., "A 3-D Ultrasound Robotic Prostate Brachytherapy System With Prostate Motion Tracking," IEEE Transactions on Robotics , vol. 28, no. 6, pp. 1382-1397, 2012.

[101] H. Tadayyon et al., "Target Motion Tracking in MRI-guided Transrectal Robotic Prostate Biopsy," IEEE Transactions on Biomedical Engineering, vol. 58, pp. 3135-3142, 2011.

[102] H. Tadayyon et al., "MRI-GUIDED prostate motion tracking by means of multislice-to-volume registration," Medical Imaging 2010: Visualization, Image-Guided Procedures, and Modeling, SPIE, vol. 7625, pp. 904 $-911,2010$.

[103] H. Xu et al., "Multi-slice-to-volume registration for MRI-guided transperineal prostate biopsy," International Journal of Computer Assisted Radiology and Surgery, vol. 10, pp. 563-572, 2014.

[104] E. Ferrante and N. Paragios, "Slice-to-volume medical image registration: A survey," Medical Image Analysis, vol. 39, pp. 101-123, 2017.

[105] K. Chinzei et al., "MR Compatible Surgical Assist Robot: System Integration and Preliminary Feasibility Study," International Conference on Medical Image Computing and Computer-Assisted Intervention, pp. 921-930, 2000

[106] S. Zangos et al.,"Mr-compatible assistance system for biopsy in a highfield-strength system: initial results in patients with suspicious prostate lesions," Radiology, vol. 259 3, pp. 903-910, 2011.

[107] M. G. Schouten et al., "Evaluation of a robotic technique for transrectal MRI-guided prostate biopsies," European Radiology, vol. 22, pp. 476 483,2011

[108] G. Miron et al., "Design and Manufacturing of Embedded Air-Muscles for a Magnetic Resonance Imaging Compatible Prostate Cancer Binary Manipulator," Journal of Mechanical Design, vol. 135, no. 1, 2012.

[109] A. Martin et al., "Robotically Guided Prostatic Fiducial Marker Insertion Under Direct MRI Guidance: A Proof of Concept in a Live Canine Subject" International Journal of Radiation Oncology • Biology - Physics, vol. 90, 2014.

[110] S. Jiang et al., "Kinematic analysis of a 5-DOF hybrid-driven MR compatible robot for minimally invasive prostatic interventions," Robotica vol. 30 7, pp. 1147-1156, 2012.

[111] S. Jiang et al., "Accurate error compensation for a MR-compatible surgical robot based on a novel kinematic calibration method," Advanced Robotics, vol. 29, pp. 1183 - 1194, 2015.

[112] S. Jiang et al., "Analysis of a novel high-precision 5-degrees of freedom magnetic resonance imaging-compatible surgery robot for needle-insertion prostate brachytherapy," Proceedings of the Institution of Mechanical Engineers, Part C: Journal of Mechanical Engineering Science, vol. 228 5, pp. 865-876, 2014.

[113] J. Cepek et al., "A system for MRI-guided transperineal delivery of needles to the prostate for focal therapy," Medical Physics, vol. 40, no. 1 , p. 012304,2013

[114] S. Jiang et al., "Design and analysis of a tendon-based MRI-compatible surgery robot for transperineal prostate needle placement," Proceedings of the Institution of Mechanical Engineers, Part C: Journal of Mechanical Engineering Science, vol. 229, no. 2, pp. 335-348, 2015.

[115] S. Eslami et al., "In-bore prostate transperineal interventions with an MRI-guided parallel manipulator: system development and preliminary evaluation," International Journal of Medical Robotics and Computer Assisted Surgery, vol. 12, no. 2, pp. 199-213, 2016.

[116] N. A. Patel et al., "System Integration and Preliminary Clinical Evaluation of a Robotic System for MRI-Guided Transperineal Prostate Biopsy," Journal of Medical Robotics Research, vol. 4 2, 2019.

[117] Y. Lin et al., "Design and Control of a Piezoelectric Actuated Prostate Intervention Robotic System*," 17th International Conference on Ubiquitous Robots (UR), pp. 175-180, 2020.

[118] L. Chen et al., "Design of a Dedicated Five Degree-of-Freedom Magnetic Resonance Imaging Compatible Robot for Image Guided Prostate Biopsy," Journal of Medical Devices, vol. 9, no. 1, pp. 015002, 2015.
[119] D. Stoianovici et al., "MRI-compatible pneumatic robot (MrBot) for prostate brachytherapy: Preclinical assessment of accuracy and execution of dosimetric plans," International Journal of Radiation Oncology • Biology • Physics, vol. 72, no. 1, pp. S306-S307, 2008.

[120] S. Jiang et al., "THE POTENTIAL FIELD-BASED TRAJECTORY PLANNING FOR NEEDLE INSERTION IN A SOFT-TISSUE MODEL," Biomedical Engineering: Applications, Basis and Communications, vol. 25, p. 1350017, 2013.

[121] P. Moreira, K. J. Boskma, and S. Misra, "Towards MRI-guided flexible needle steering using fiber bragg grating-based tip tracking," 2017 IEEE International Conference on Robotics and Automation (ICRA), pp. 4849-4854, 2017.

[122] J. Bohren, I. Iordachita, and L. L. Whitcomb, "Design requirements and feasibility study for a 3-DOF MRI-compatible robotic device for MRIguided prostate intervention," 2012 IEEE International Conference on Robotics and Automation, pp. 677-682, 2012.

[123] J. A. M. Cunha et al., "Toward adaptive stereotactic robotic brachytherapy for prostate cancer: demonstration of an adaptive workflow incorporating inverse planning and an MR stealth robot," Minimally Invasive Therapy \& Allied Technologies, vol. 19, pp. 189 - 202, 2010.

[124] M. Borot de Battisti et al., "A novel adaptive needle insertion sequencing for robotic, single needle MR-guided high-dose-rate prostate brachytherapy," Physics in Medicine \& Biology, vol. 62 10, pp. 40314045, 2017.

[125] J. Tokuda et al., "Preclinical evaluation of an MRI-compatible pneumatic robot for angulated needle placement in transperineal prostate interventions," International Journal of Computer Assisted Radiology and Surgery, vol. 7, no. 6, pp. 949-957, 2012.

[126] W. Ji et al., "Reconfigurable Fiducial-Integrated Modular Needle Driver for MRI-Guided Percutaneous Interventions," Journal of Medical Devices-Transactions of The ASME, vol. 7, p. 030915, 2013.

[127] N. A. Patel et al., "Closed-loop asymmetric-tip needle steering under continuous intraoperative MRI guidance," 2015 37th Annual International Conference of the IEEE Engineering in Medicine and Biology Society (EMBC), pp. 4869-4874, 2015.

[128] P. Zamecnik et al., "Automated real-time needle-guide tracking for fast 3-T MR-guided transrectal prostate biopsy: a feasibility study," Radiology, vol. 273 3, pp. 879-86, 2014.

[129] S. Jiang, X. Liu, and Y. Song, "3D Trajectory Planning Based on FEM with Application of Brachytherapy," $2^{\text {nd }}$ International Conference on Biomedical Engineering and Informatics, pp. 1-5, 2009.

[130] M. Barral et al., "In-Bore Transrectal MRI-Guided Biopsy With Robotic Assistance in the Diagnosis of Prostate Cancer: An Analysis of 57 Patients." AJR. American Journal of Roentgenology, pp. 1-9, 2019.

[131] G. R. Cho, S. T. Kim, and J. Kim, "Backlash Compensation for Accurate Control of Biopsy Needle Manipulators having Long Cable Transmission," International Journal of Precision Engineering and Manufacturing, vol. 19, pp. 675-684, 2018.

[132] P. Moreira and S. Misra, "Biomechanics-Based Curvature Estimation for Ultrasound-guided Flexible Needle Steering in Biological Tissues," Annals of Biomedical Engineering, vol. 43, pp. 1716-1726, 2014.

[133] C. Ménard, J.-F. Pambrun, and S. Kadoury, "The utilization of magnetic resonance imaging in the operating room," Brachytherapy, vol. 16 4, pp. 754-760, 2017

[134] M. Rivard, Dee-Ann. Radford Evans and I. Kay, "A technical evaluation of the Nucletron FIRST system: Conformance of a remote afterloading brachytherapy seed implantation system to manufacturer specifications and AAPM Task Group report recommendations" Brachytherapy, vol. 6, pp. $22-50,2005$.

[135] R. Merzouki et al., "Autonomous guidance system for needle-holding equipment," PCT/FR2016/051783, WO/2017/009572, 2017.

[136] M. Sato et al., "Remote radioactive seed-loading device for permanent brachytherapy of oral cancer with Au-198 grains," ROBOMECH Journal, vol. 4, pp. 1-10, 2017

[137] J. A. Proffitt and A. K. Ball, "A Device for the Automated Loading and Detection of Brachytherapy Elements Using Nonmechanical Methods for use in Prostate Cancer Treatment," Journal of Medical DevicesTransactions of The ASME, vol. 6, pp. 021009, 2012.

[138] Y. Chen et al., "Stereotactic systems for MRI-guided neurosurgeries: A state-of-the-art review," Annals of Biomedical Engineering, vol. 47, pp. 335-353, 2018.

[139] N. Hata et al., "MR Imaging-guided Prostate Biopsy with Surgical Navigation Software: Device Validation and Feasibility," Radiology, vol. 2201 , pp. 263-8, 2001.

[140] J. M. Albert et al., "Magnetic resonance imaging-based treatment planning for prostate brachytherapy" Brachytherapy, vol. 12 1, pp.30-7, 2013. 
[141] J. Ghelfi et al., "Evaluation of the Needle Positioning Accuracy of a Light Puncture Robot Under MRI Guidance: Results of a Clinical Trial on Healthy Volunteers," CardioVascular and Interventional Radiology, vol. 41, pp. 1428-1435, 2018.

[142] T. Penzkofer et al., "How MRI compatible is "MRI compatible"? A systematic comparison of artifacts caused by biopsy needles at 3.0 and 1.5 T," CardioVascular and Interventional Radiology, vol. 36, pp. 16461657,2013

[143] W. Wang et al., "Real-time active MR-tracking of metallic stylets in MR-guided radiation therapy," Magnetic Resonance in Medicine, vol. 73 5, pp. 1803-11, 2015.

[144] N. Kuo et al., "MRI-based prostate brachytherapy seed localization," 2010 IEEE International Symposium on Biomedical Imaging: From Nano to Macro, April 2010, pp. 1397-1400.

[145] VP. J. Panagiotopoulos, "Webb S: Contemporary IMRT: Developing Physics and Clinical Implementation. Series in Medical Physics and Biomedical Engineering," BioMedical Engineering OnLine, vol. 4, pp. 20, 2005.

[146] T. Bortfeld, "IMRT: a review and preview." Physics in Medicine \& Biology, vol. 51 13, pp. R363-79, 2006.

[147] M. Hussein et al. "Automation in intensity modulated radiotherapy treatment planning-a review of recent innovations," The British Journal of Radiology, vol. 91 1092, pp. 20180270, 2018

[148] M. Wang et al.,"A Review on Application of Deep Learning Algorithms in External Beam Radiotherapy Automated Treatment Planning," Frontiers in Oncology, vol. 10, 2020.

[149] S. P. Register et al., "An MRI-based dose-reponse analysis of urinary sphincter dose and urinary morbidity after brachytherapy for prostate cancer in a phase II prospective trial," Brachytherapy, vol. 12 3, pp. 210-6, 2013.

[150] R. Nath et al., "Dosimetry of interstitial brachytherapy sources: recommendations of the AAPM Radiation Therapy Committee Task Group no. 43. American Association of Physicists in Medicine," Medical Physics, vol. 22 2, pp. 209-34, 1995.

[151] L. Beaulieu et al., "Report of the Task Group 186 on model-based dose calculation methods in brachytherapy beyond the TG-43 formalism: current status and recommendations for clinical implementation" Medical Physics, vol. 39 10, pp. 6208-36, 2012.

[152] S. Enger et al., "Model-Based Dose Calculation Algorithms for Brachytherapy Dosimetry," Seminars in Radiation Oncology, vol. 30 1, pp. 77-86, 2020

[153] K. B. B. Boni et al., "MR to CT synthesis with multicenter data in the pelvic era using a conditional generative adversarial network," Physics in Medicine \& Biology, 2020.

[154] H. Hu, Y. Shao, and S. Hu, "A Review of the Application of Deep Learning in Brachytherapy," Open Access Library Journal, vol. 7, pp. $1-9,2020$

[155] X. Mao et al., "RapidBrachyDL: Rapid Radiation Dose Calculations in Brachytherapy Via Deep Learning," International Journal of Radiation Oncology • Biology • Physics, 2020.

[156] M. Moerland et al., "Evaluation of permanent I-125 prostate implants using radiography and magnetic resonance imaging" International Journal of Radiation Oncology • Biology • Physics, vol. 37 4, pp. 927-33, 1997.

[157] R. A. Cormack et al., "A clinical method for real-time dosimetric guidance of transperineal 125I prostate implants using interventional magnetic resonance imaging" International Journal of Radiation Oncology • Biology • Physics, vol. 46 1, pp. 207-14, 2000.

[158] A. Tejwani et al.,"Case series analysis of post-brachytherapy prostate edema and its relevance to post-implant dosimetry. Post-implant prostate edema and dosimetry" Journal of Contemporary Brachytherapy, vol. 4, pp.75 - 80, 2012.

[159] H. Westendorp et al.,"Edema and Seed Displacements Affect Intraoperative Permanent Prostate Brachytherapy Dosimetry," International Journal of Radiation Oncology • Biology • Physics, vol. 96 1, pp.197205, 2016.

[160] K. A. Mountris et al.,"Modeling the impact of prostate edema on LDR brachytherapy: a Monte Carlo dosimetry study based on a 3D biphasic finite element biomechanical model," Physics in Medicine \& Biology, vol. 62 6, pp.2087-2102, 2017.

[161] N. Harland and A. Stenzl,"Micro-Ultrasound: a way to bring imaging for prostate cancer back to urology," Prostate International, 2021

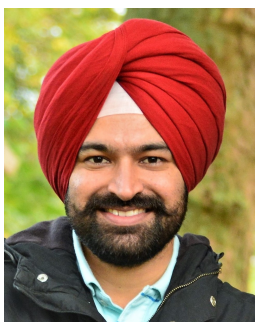

Sepaldeep Singh Dhaliwal is currently an engineer at the Laboratory CRIStAL UMR-CNRS 9189, Univ of Lille, FR. He received a Master's in Robotics (2018) from Polytech \& Ecole Centrale de Lille, FR. Master's in Industrial Design (2015) from PEC Univ of Technology Chandigarh, and Bachelor's in Mechanical Engg. (Punjabi Uni), India. In 2016-17, he worked as Research Fellow at $\mathrm{CoE}$ in Industrial \& Product Design, Chandigarh. His main interests are in medical robotics and product development.

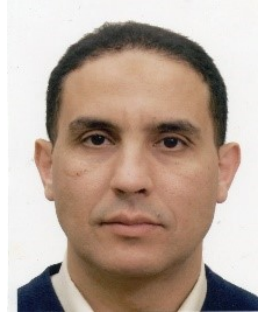

Taha Chettibi received a Ph.D. degree in robotics from Polytechnic school (EMP) of Algiers, Algeria, in 2006. He is currently a lecturer in mechanical engineering at the department of mechanical engineering at BLIDA-1 University (Algeria). His major research focus is robotic systems modelling, trajectory planning and design optimization of mechanical systems. In 2018, He was a visiting Professor at Laboratory CRIStAL UMR-CNRS 9189, University of Lille, France.

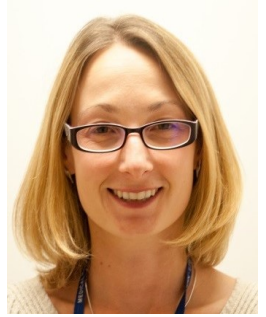

Sarah Wilby is currently a principal clinical scientist at Portsmouth Hospital University NHS Trust. She completed an MSc at the University of Surrey before becoming a Chartered Scientist at University Hospital Southampton. Sarah spent several years in industry as a clinical support specialist in radiotherapy. In 2018 she joined the CoBra EU-project in robotic brachytherapy and is working on a $\mathrm{PhD}$ in phantom design.

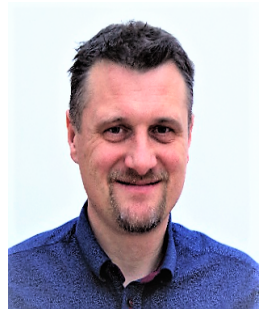

Wojciech Polak is currently a Medical Physics Expert, Head of Brachytherapy, Principal Clinical Scientist at Portsmouth Hospital Univ NHS Trust, and an Honorary Lecturer at the Univ Portsmouth. Wojciech completed an MSc in Physics in 2000 at Jagiellonian Univ, Kraków, specializing in Medical Physics. He obtained a Ph.D. (2006) in Physics at the Institute of Nuclear Physics PAN, Kraków. In 2012, completed the IPEM Medical Physics training in Radiotherapy \& registered as a Healthcare Scientist.

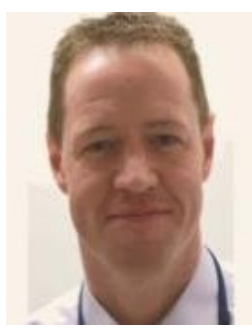

Antony L. Palmer is currently the Lead Scientist for Medical Physics at Portsmouth Hospitals University NHS Trust. Tony obtained a Ph.D. in brachytherapy dosimetry at the University of Surrey in 2015. He is head of Radiotherapy Physics at Portsmouth Hospitals and holds visiting research appointments at the University of Surrey, University College London, and Portsmouth University.

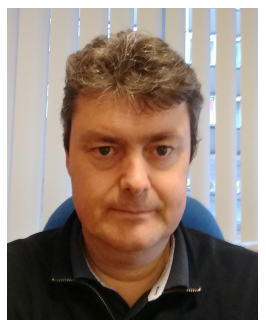

Nick Reynaert is currently the head of the medical physics department at the Jules Bordet Institute in Brussels, overseeing the imaging, nuclear medicine, and radiotherapy departments. He is also the director of the Medical Physics Laboratory at the ULB (Free Brussels University) and responsible for a number of academic courses. He obtained his Ph.D. from the Ghent University (Belgium) in 2000 and his HDR from the University of Lille (France) in 2017.

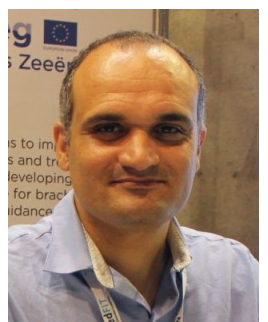

Rochdi Merzouki is a Professor in Robotics an Mechatronics at CRIStAL UMR-CNRS 9189 Univ of Lille. He received a Ph.D. degree in Autonomous Robotics from the Univ of Versailles, FR, in 2002. $\mathrm{He}$ is the contact point of the regional AIP-Primeca network of Robotics in Polytech'Lille. He was the coordinator of the EU-project InTraDE'09-15 and project manager of EU-project CoBra'18-22 His main research interests concern the system of systems engg, modeling, and supervision of mechatronics systems applied to autonomous and soft robotics. 